\title{
PHASE-REFERENCED INTERFEROMETRY AND NARROW-ANGLE ASTROMETRY WITH SUSI
}

\author{
Y. KOK*,§, M. J. IRELAND ${ }^{\dagger, \ddagger}$, P. G. TUTHILL*, J. G. ROBERTSON*, \\ B. A. WARRINGTON ${ }^{\dagger}$, A. C. RIZZUTO ${ }^{\dagger}$ and W. J. TANGO* \\ *Sydney Institute for Astronomy \\ School of Physics \\ The University of Sydney \\ NSW 2006, Australia \\ †Department of Physics and Astronomy \\ Macquarie University \\ NSW 2109, Australia \\ ${ }^{\ddagger}$ Australian Astronomical Observatory \\ PO Box 915, North Ryde \\ NSW 1670, Australia \\ §y.kok@physics.usyd.edu.au
}

Received 2013 July 1; Revised 2013 September 22; Accepted 2013 November 28; Published 2014 January 6

\begin{abstract}
The Sydney University Stellar Interferometer (SUSI) now incorporates a new beam combiner, called the Microarc-second University of Sydney Companion Astrometry instrument (MUSCA), for the purpose of high precision differential astrometry of bright binary stars. Operating in the visible wavelength regime where photon-counting and post-processing fringe tracking is possible, MUSCA will be used in tandem with SUSI's primary beam combiner, Precision Astronomical Visible Observations (PAVO), to record high spatial resolution fringes and thereby measure the separation of fringe packets of binary stars. In its current phase of development, the dual beam combiner configuration has successfully demonstrated for the first time a dual-star phase-referencing operation in visible wavelengths. This paper describes the beam combiner optics and hardware, the network of metrology systems employed to measure every noncommon path between the two beam combiners and also reports on a recent narrow-angle astrometric observation of $\delta$ Orionis A (HR 1852) as the project enters its on-sky testing phase.
\end{abstract}

Keywords: Optical long baseline interferometry, visible wavelengths, metrology, data reduction algorithm, binary stars.

\section{Introduction}

Phase-referencing in interferometry is a technique which uses the phase information in the interference signal of a reference object to correct the phase of the signal from a target object for path length fluctuations in the atmosphere. The target can either be the same or a nearby object. This technique has been widely used in radio interferometry but its potential in optical long baseline interferometry was only realized in the early 1990s (Shao \& Colavita, 1992; Colavita, 1992). This technique is mainly used (1) to extend the effective atmospheric coherence time beyond the limit imposed by atmospheric turbulence in order to increase the sensitivity of an interferometer (Quirrenbach et al., 1994; Hummel et al., 2003; Lane \& Colavita, 2003) and (2) to measure the phase difference between the target and the reference signal in order to determine the relative position of the target object in the sky (Lane \& Muterspaugh, 2004; Delplancke, 2008; Woillez et al., 2010).

Unlike radio interferometry where phasereferencing can be achieved by rapidly slewing the antenna back and forth between the target object and the reference, in optical interferometry both objects must be observed simultaneously because 
the atmospheric coherence time scale is measured in milliseconds. Therefore, phase-referencing optical interferometers usually have two beam combiners to record interference signals from two objects at the same time. A dual beam combiner for an optical interferometer is analogous to an adaptive optics (AO) instrument for a single mirror imaging telescope. The first dual beam combiner setup for an optical long baseline interferometer was installed at the Palomar Testbed Interferometer (PTI). The beam combiners operated in the near IR wavelengths ( $\mathrm{K}$ band) and were designed to perform phase-referenced narrow-angle astrometry (Colavita et al., 1999).

With similar scientific motivation, a second beam combiner was recently installed at the Sydney University Stellar Interferometer (SUSI) (Davis et al., 1999a). The new setup at SUSI aims to perform phase-referenced narrow-angle astrometry as a mean to search for extra-solar planets around bright close binary stars. Despite the similar goal, the implementation of the dual beam combiner configuration at SUSI as compared to PTI is vastly different. The beam combiners at SUSI operate at visible wavelengths $(0.77-0.9 \mu \mathrm{m})$ and are unconventional in that they perform dual-star phasereferencing in post-processing. This offers the beam combiners at SUSI the potential of measuring the relative position of a target object about three times more precisely at the same baseline at a cost of tighter tolerances, and a method to perform real time group-delay tracking at much lower bandwidth. The achievable astrometric precision, which is limited by the phase uncertainty of the interference signal, is proportional to the mean wavelength of the signal obtained with the beam combiners. A shorter wavelength translates to a higher astrometric precision. The mean wavelength in which the beam combiners in SUSI are operating at is $\sim 3 \times$ of $\mathrm{K}$ band (i.e. $\sim 2.2 \mu \mathrm{m}$ ). For this same reason, the phase information of the reference signal must be accurately known to a fraction of the mean wavelength (i.e. a tighter tolerance with shorter wavelength) so that the phase uncertainty of interference signal of the target object as a result of the phase-referencing process is small and the visibility of the fringe packet is not lost due to incoherent integration.

The optical setup of the beam combiners at SUSI is described in the following section. Then, the approach to phase-referencing via a network of metrology systems and the details of the data processing required are discussed in Secs. 3 and 4 respectively. Sample results from recent test observations are then presented and discussed in Secs. 5 and 6 . Finally, a summary of future work to be performed and scientific goals of the instruments are discussed in Sec. 7.

\section{Instrument Configuration}

SUSI is a 2-element optical long baseline interferometer located near Narrabri, New South Wales, Australia. Figure 1 shows a schematic diagram of such an interferometer. Starlight collected from 2 siderostats (labeled as apertures in Fig. 1) are reflected by a series of mirrors into one or more light combining instruments. SUSI has a configurable (5-160 m) North-South oriented baseline (distance between 2 siderostats, commonly denoted as $\vec{B}$ or $B$ ). It was completed in 1991 and for over two decades, the subsystem instruments within the

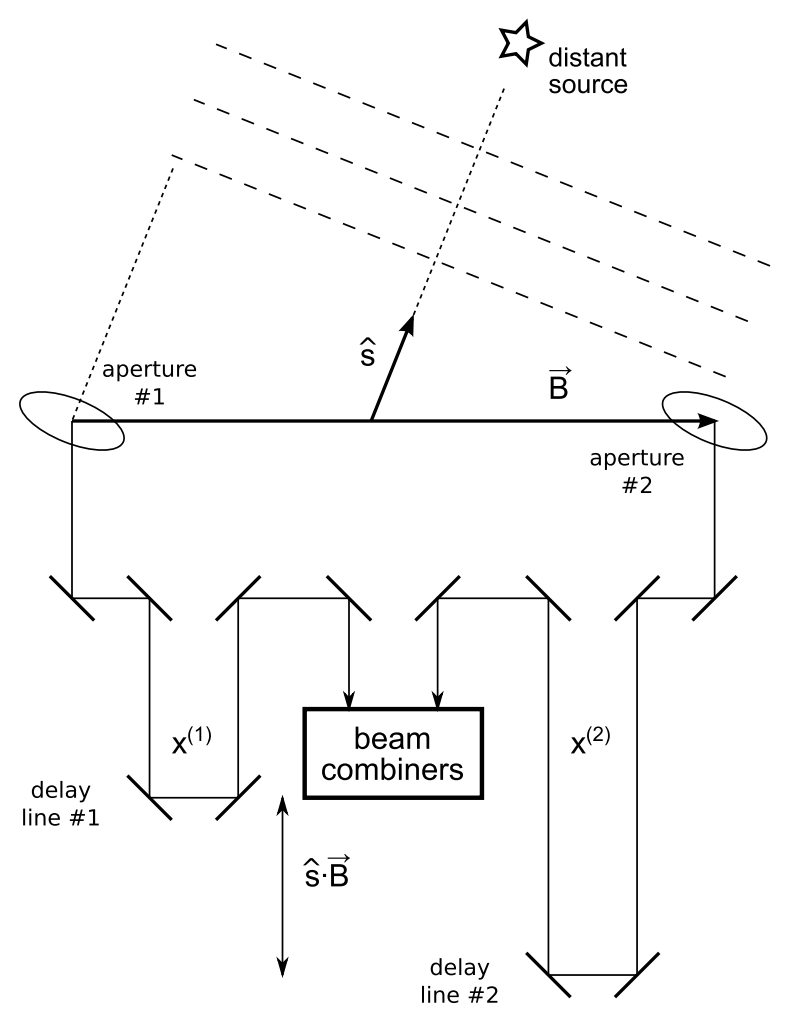

Fig. 1. A minimalist 2-element optical long baseline interferometer. The baseline, $\vec{B}$, defines the separation between the 2 light collecting elements of the interferometer while the unit vector $\hat{s}$ (commonly known as the pointing vector) describes the angle between a star of interest and the baseline. The optical path length of each delay line is denoted by the symbol $X$. Interference fringes are formed when the OPD between the delay lines is $\approx \hat{s} \cdot \vec{B}$. 
interferometer, especially the beam combiners, have been constantly upgraded to increase its sensitivity and capability (ten Brummelaar \& Tango, 1994; Lawson, 1994; Davis et al., 2007; Robertson et al., 2012). The current main beam combiner in SUSI is the Precision Astronomical Visible Observation (PAVO) instrument (Ireland et al., 2008) which has been operating since 2009 and has been used mainly for stellar diameter measurements and astrometry of binary stars (Antoci et al., 2013; Rizzuto et al., 2013). Joining it is the recently installed Microarcsecond University of Sydney Companion Astrometry (MUSCA) beam combiner instrument. MUSCA is designed to operate alongside PAVO for high precision narrow-angle astrometry.

The two beam combiners are fed with a pair of starlight beams from the two arms of the interferometer but they each use a slightly different part of the visible light spectrum. They share the same main delay line for the tracking of their interference signals in the form of interference fringes which are only visible when the optical path difference (OPD) between the two arms of the interferometer is near zero (within the coherence length of the starlight whose range of wavelengths are usually defined by a bandpass filter). Such interference signal is usually known as a fringe packet (see Fig. 2 for examples). The main delay line is made up of 2 folded optical paths, one for each arm. Both paths, represented by the structures labeled "delay line \#1" and "delay line \#2" in Fig. 1, are simultaneously controlled to differentially add optical path to one arm and remove the same amount from the other. Detail description of the main delay line is given by Davis et al. (1999a). In addition, MUSCA has a differential delay line (DDL) for switching between fringe packets of two stars in a binary system. The DDL is needed because the optical path modulation used to sweep through a fringe packet in MUSCA is often smaller than the typical optical path length between peaks of the envelope of the fringe packets of two stars in a binary system. The limit of the optical path modulation is illustrated by the width of the rectangular window around each fringe packet in Fig. 2 while the fringe packet separation, which is the main observable of the PAVO+MUSCA dual beam combiner configuration, is represented by the dimension $S$ in the figure. Suppose the phase delay of a fringe packet, denoted by the symbol $m_{1}$ or $m_{2}$, is defined as the OPD between two arms of the interferometer computed at the mid-range of the optical path modulation in MUSCA, then a measurement of the displacement of the DDL, denoted by the symbol $d$, and the difference in phase delay between the pair of stellar fringe packets yield the fringe packet separation, $S$. The relation between $S$ and the on-sky separation of the two stars is given as,

$$
S=\left(\hat{s}_{2}-\hat{s}_{1}\right) \cdot \vec{B}
$$

where $\hat{s}_{1}$ or $\hat{s}_{2}$ is a unit vector that describes the angle between a star and the baseline of the interferometer (see Fig. 1).

During observations, PAVO is used as a groupdelay tracker to keep the fringe packet of a star within the modulation range of optical path in MUSCA. Then in post-processing, PAVO and a network of interferometer-based metrology systems are used to estimate the OPD at each optical path modulation step in the MUSCA. PAVO observes only one reference fringe packet (usually the one of the primary star in a binary system) throughout an observation session but MUSCA alternately

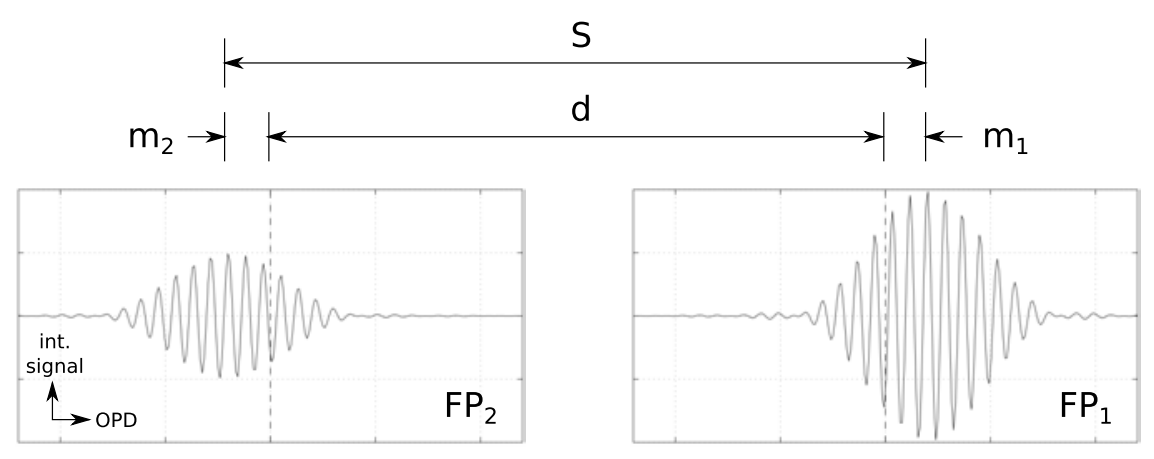

Fig. 2. The interference signal of two nearby stars. Due to the range of optical modulation, represented by the width of the rectangular window, often only one separated fringe packet (FP) is visible to MUSCA at a given time. The selection of a fringe packet to be observed is controlled by the position of the differential delay line (DDL) in MUSCA. The separation of the fringe packets can be estimated from the measurement of the displacement of the DDL, $d$, and the phase delay difference of the fringe packets, $m_{2}-m_{1}$. 
observes either one of the two fringe packets by toggling the position of the DDL every $5-15$ min depending on the atmospheric seeing conditions. The relative position of the fringe packets is measured simultaneously but indirectly because as PAVO tracks the reference (primary) fringe packet MUSCA observes the secondary. The reference phase of the reference fringe packet is assumed to be stable over time. The stability of the reference fringe packet is evaluated and discussed in Sec. 5.3. With a typical integration time of $10-15$ min on the fringe packets (during dual-star phase-referencing mode), the astrometric precision due to anisoplanatism of the atmospheric turbulence (Shao \& Colavita, 1992) for star separation of $<1^{\prime \prime}$ is negligible $(<10 \mu \mathrm{as})$ as compared to the reference phase stability (refer to Sec. 5 for detail discussion). This assumes a baseline of $>60 \mathrm{~m}$ is used and the atmospheric scaling factor at SUSI is $\sim 780 \mathrm{~m}^{2 / 3} \mathrm{~s}^{1 / 2}$ arcsec.

\subsection{PAVO}

PAVO is a multi-axially aligned Fizeau-type interferometer, but unlike a typical Fizeau interferometer, PAVO forms spatially modulated interference fringes in a pupil plane and then spectrally disperses the fringes. It also employs spatial filtering in its image plane and an array of cylindrical lenslets to utilize the full multi- $r_{0}$ aperture of the siderostats at SUSI. A similar setup (Ireland et al., 2008) is operational at the Center for High Angular Resolution Astronomy (CHARA) array. Figure 3 shows the schematic diagram of the PAVO beam combiner.

The actual optical setup is divided into two parts: a front-end and a back-end arrangement. The front-end optics splits the incoming beams into various components while the back-end optics (Fig. 3) recombines the beams. Figure 4 shows

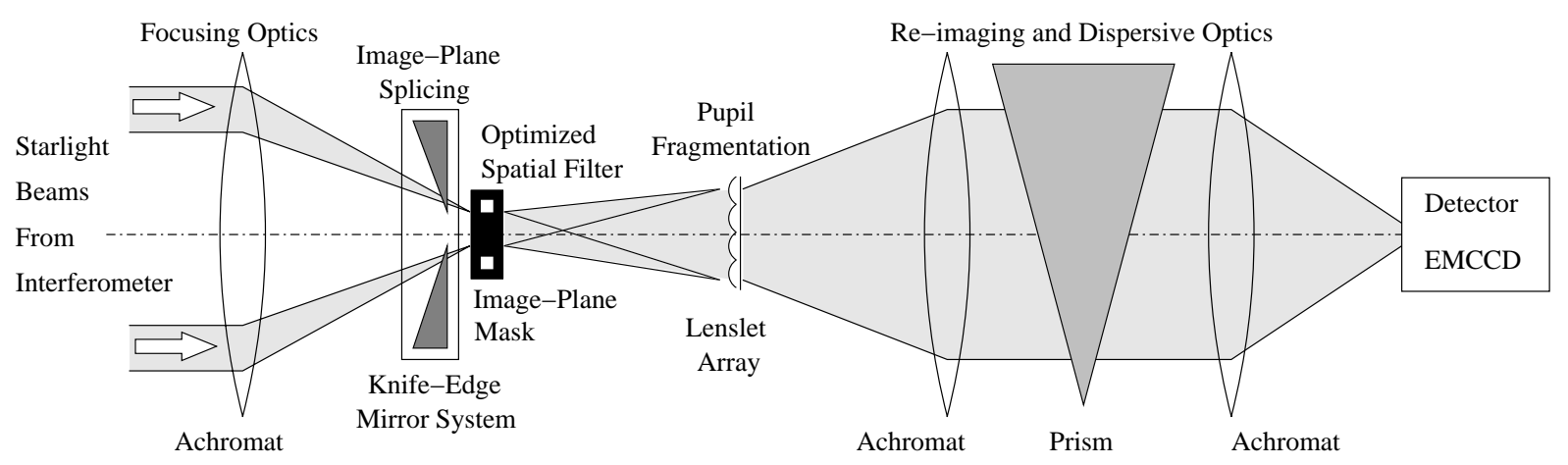

Fig. 3. Schematic diagram of the PAVO beam combiner at SUSI.

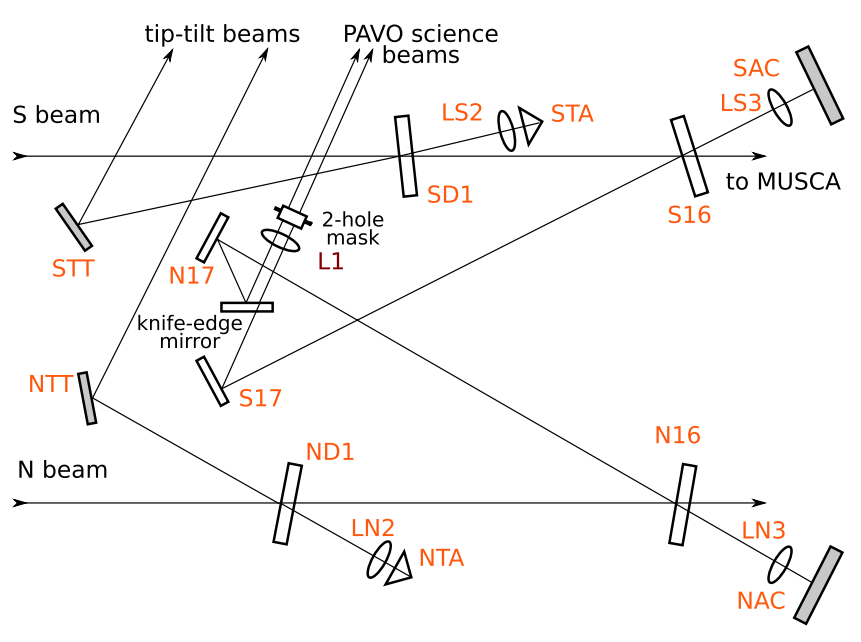

(a)

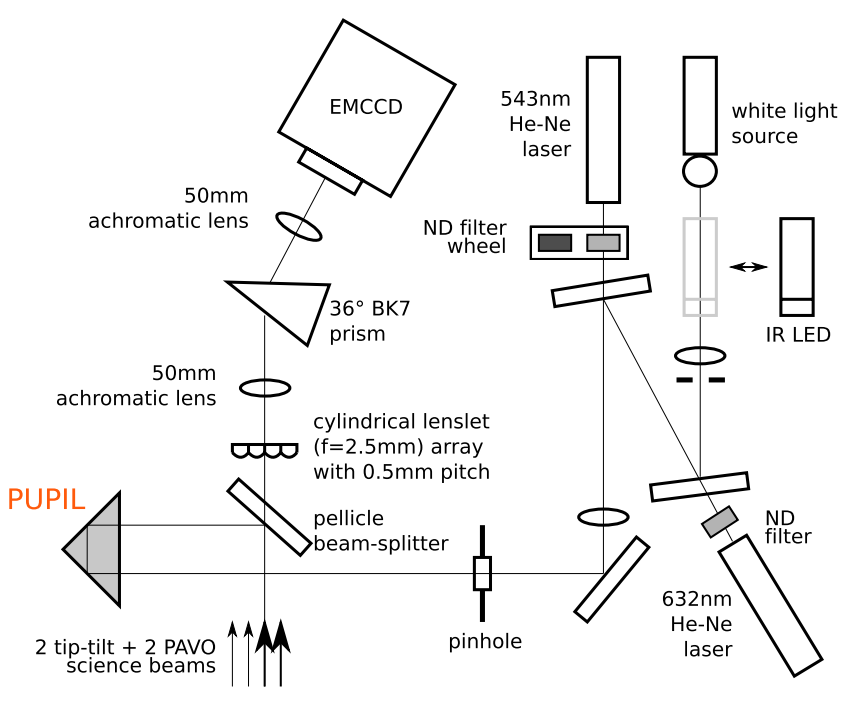

(b)

Fig. 4. The (a) front- and (b) back-end optical setup of PAVO in the laboratory. 
the overall arrangement of the PAVO optics. Figure 4(b) also shows the location of the metrology sources which are discussed in Sec. 3.

The North (N) and South (S) beams from the siderostats propagate from the left to right in Fig. 4(a). A pair of dichroic filters, ND1 and SD1, separate the tip-tilt from the science beams. The filters reflect light of wavelength shorter than $0.54 \mu \mathrm{m}$ and transmit light of longer wavelengths. The reflected blue end of the visible spectrum is fed into a tip-tilt system which acts as a first order AO system that removes the tip and tilt components (or the phase ramp) from the wavefront. The science beams are then separated by wavelength into 2 parts, one for PAVO and another for MUSCA, by another pair of dichroic filters, N16 and S16. These filters reflect light of wavelength shorter than $0.79 \mu \mathrm{m}$ and transmit light of longer wavelengths. As a consequence, the middle part of the visible spectrum $(0.54-0.79 \mu \mathrm{m})$ is reflected into PAVO while the red end of the spectrum is transmitted into MUSCA.

The beams are actually converging $(\sim f / 700)$ because there is a focusing lens in each arm of the interferometer (not shown in Fig. 4) which focuses to an image plane located at the PAVO two-hole mask. Each of the $\sim 1.2 \mathrm{~mm}$ apertures in the mask corresponds to a FOV of $\sim 2^{\prime \prime}$ on the sky. The holes are separated by twice their width and are used as low-pass spatial filters to clean aberrated images of stars. A knife-edge mirror is used to align the beams through the apertures of the mask.

The beams are aligned at the pupil plane to form spatially modulated fringes. Fringes are formed in the pupil plane so that they are less sensitive to seeing and misalignment. At the pupil plane, different parts of the combined pupil are sampled by an array of lenslets that act as virtual slits across the image plane for a spectrograph. The spectrograph, which consists of a BK7 prism and a pair of six-element 50-mm lenses, spectrally disperses the fringes before they are re-imaged onto an EMCCD camera.

More in-depth description of PAVO at SUSI can be found in several other papers (Tuthill et al., 2008; Robertson et al., 2010, 2012).

\subsection{MUSCA}

MUSCA is a pupil-plane Michelson interferometer which operates at wavelengths between $0.77 \mu \mathrm{m}$ and $0.91 \mu \mathrm{m}$. It forms temporal fringes by modulating

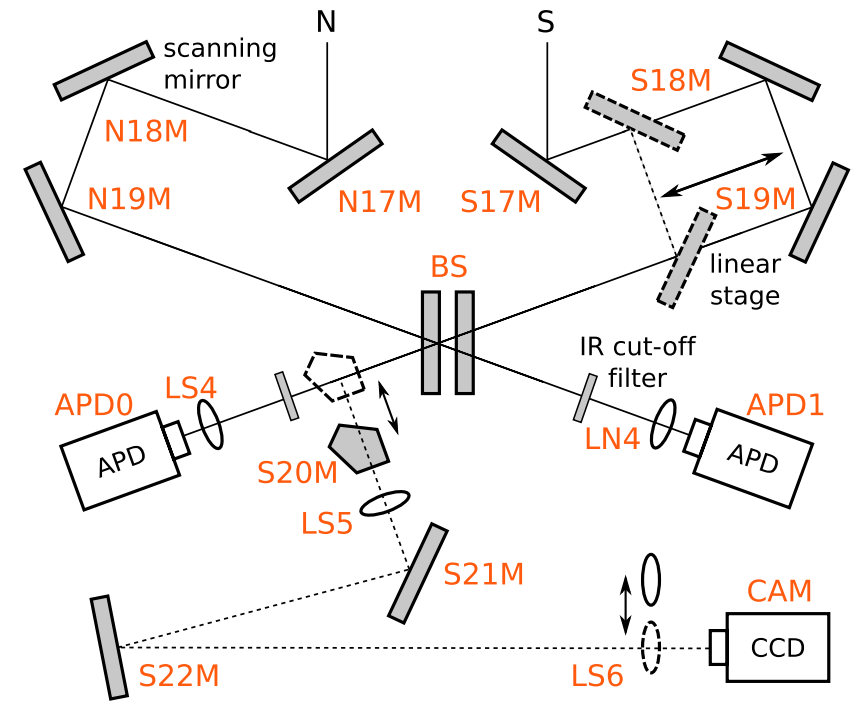

Fig. 5. Optical setup of MUSCA.

the optical path length of one arm with time. Figure 5 shows the optical path and components of MUSCA. Incoming converging light beams from the top of the figure continue from Fig. 4(a).

The beams are combined co-axially at the beam-splitter, BS. A scanning mirror $\mathrm{N} 18 \mathrm{M}$ is used to modulate the optical path length of the North arm of MUSCA. It is attached to a piezoelectric transducer (PZT) which converts voltage to physical displacement. Voltage is applied in small steps and at an interval of about $0.2-0.3 \mathrm{~ms}$ per step. In order to avoid excitation of unnecessary mechanical resonance the modulation signal is passed through a 1-ms low-pass RC filter before it is fed to the transducer. The intensity variation of the combined beams as a result of the optical path length modulation is recorded as temporal fringes using a pair of SPCM-AQR-14-FC selfcontained single photon counting avalanche photodiode (APD) modules from PerkinElmer.

The maximum path length modulation attainable from the transducer is $\sim 140 \mu \mathrm{m}$ (maximum throw of the PZT is $\sim 100 \mu \mathrm{m}$ in a folded optical path) but the typical value used for MUSCA is $\sim 25 \mu \mathrm{m}$. This is chosen for MUSCA because (1) it matches with the PAVO's group delay tracking window and (2) it is approximately $2 \times$ larger than the expected width of the MUSCA fringe packet. Making the range larger is undesirable because the PAVO group delay estimates are unreliable beyond its tracking window and therefore phase-referencing is futile at that range. 
A linear translational stage (T-LS28 from Zaber Technologies) and two mirrors on the South arm of MUSCA form the DDL for the beam combiner. It is used for fringe finding by equalizing the optical path length between the North and South arms. The fiducial location where OPD between the arms is defined to be zero is chosen at the middle of the scan range of the scanning mirror. As fringe packets from two different stars are separated in optical path length the DDL must alternate between two positions in order to place either one of the fringe packets into the scan window. Effectively the DDL is used to 'select' one fringe packet for observation at any one time.

The stage is stepper-motor driven and has a built-in open loop position control system. The stepper motor converts rotary motion into linear motion via a leadscrew. The total travel distance of the stage is more than $8 \mathrm{~mm}$, which is the required span to observe binary star separations as wide as $10^{\prime \prime}$ using a 160-m baseline. At an average slew speed of $\sim 2 \mathrm{~mm} / \mathrm{s}$, it takes the stage less than $5 \mathrm{~s}$ to cover that distance. The leadscrew-based open loop position control system of the stage, which has a nominal accuracy of $12 \mu \mathrm{m}$ and a repeatability of $<0.4 \mu \mathrm{m}$, is not the only mechanism put in place to measure the position of the stage for MUSCA's astrometric application. It is also being measured by a dual laser metrology system which has a precision better than $5 \mathrm{~nm}$. The metrology system works hand in hand with the open loop position control system and is discussed in detail in a separate paper (Kok et al., 2013a).

The role of each field lens, LN4 and LS4 $(f=$ $35 \mathrm{~mm}$ ) is to ensure that the pupil of any offaxis star (in MUSCA's case, the secondary of a binary system) is formed within the active (photosensitive) region of an APD. Otherwise MUSCA will not be able to detect fringes from stars further than $0.2^{\prime \prime}$ from the on-axis reference star.

The IR cut-off filters in front of each APD are intended to reduce the number of background photon counts originating from an IR metrology laser $(\lambda=1.152591 \mu \mathrm{m})$ which is used with the main delay line at SUSI. The filter cut-off wavelength is about $1 \mu \mathrm{m}$.

\subsection{Optical alignment}

Alignment light sources in SUSI can be divided into two sets according to their function. The first set consists of a laser and a bright halogen lamp and is used for general optical alignment and internal fringe searching. They are injected into the optical path in front of PAVO (see Fig. 4(b)) through a pin hole and a pellicle. The second set consists of LEDs and is used to align the narrow-angle baseline of MUSCA with the wide-angle baseline of SUSI. The LEDs are attached precisely in the center of the back of the mirror mount at every siderostat. The alignment process takes advantage of the imperfection in the dichroic filter fabrication, e.g. the finite slope of the filter profile at the cut-off wavelengths, to reflect light back into the beam combiners. PAVO uses its EMCCD camera for optical alignment but MUSCA uses a separate CCD camera specifically for the same purpose. In order to use the latter, a prism is slid into the optical path during alignment and is removed during observation.

The wide-angle baseline of an interferometer is defined as a vector between pivot points of two siderostats. The pivot point of a siderostat is a virtual point where the two rotational (elevation and azimuth) axes of the siderostat intersect and typically coincides with the center of the siderostat mirror. The wide-angle baseline vector is obtained by observing stars separated by large angles and located in various parts of the sky, noting the differences between the expected and the actual optical delays (offsets) required to produce stellar fringes based on a set of estimated dimensions and finally solving the simultaneous equations of offsets to obtain a correction to the estimated baseline vector initially used. The uncertainty of the wide-angle baseline obtained with this method is in the regime of several micrometers in principle, and $\sim 100 \mu \mathrm{m}$ for SUSI once all errors are taken into account. The baseline vector only needs to be updated on a time-scale of the order of a few weeks if the accuracy of its value is to remain better than $50 \mu \mathrm{m}$ (Davis et al., 1999b). On the other hand, the narrow-angle baseline of the same interferometer (also known as the imaging baseline according to a definition by Woillez \& Lacour (2013)) is defined as a vector between the center of two entrance pupils of a beam combiner projected onto the siderostats. The following is an example to easily understand the relation between the wide- and narrow-angle baselines. Suppose a two-hole aperture-mask is put over each siderostat mirror, fringes from each pair of apertures (one from each siderostat) can be used to make astrometric measurements of a binary star on a slightly different narrow-angle 
baseline simultaneously, despite the aperture pairs between the two masks share the same pivot points, which defines the wide-angle baseline of the two interferometers.

If the wide- and narrow-angle baselines are not co-aligned, then using the wide-angle baseline solution for narrow-angle astrometry of a close binary star system is erroneous which then systematically limits the precision of the astrometric measurement. In order to achieve a precision of $10 \mu \mathrm{as}$, the co-alignment between the two baselines must be better than $1 \mathrm{~mm}$ per $100 \mathrm{~m}$ baseline for a binary separation of $>1^{\prime \prime}$. The effect of baseline uncertainty on the astrometric precision of the measurement has been extensively discussed elsewhere (Muterspaugh et al., 2010; Woillez \& Lacour, 2013).

The image of an LED at a siderostat formed on the MUSCA camera has a diameter of about 12 pixels, where $\sim 1$ camera pixel in the image plane corresponds to a dimension of $1 \mathrm{~mm}$ at the siderostats. Therefore, in order to meet the baseline uncertainty requirement, the centroid of the LED images from the North and South siderostats must be aligned to within 1 pixel. The centroid of an LED image is computed by fitting a two-dimensional Gaussian model to the image in real time while mirrors are being adjusted for alignment. At the same time, the LED images are also aligned to the fiducial center of the tip-tilt system so that the tip-tilt beams have the same optical axis as the MUSCA science beams. This alignment procedure is carried out every night before an astrometric observation with PAVO and MUSCA commences.

\section{Metrology Systems}

There are 4 metrology systems in SUSI which are designed to measure the OPD of different parts of the interferometer. The main metrology is a double laser homodyne interferometer and operates at an IR wavelength of $1.152591 \mu \mathrm{m}$ with a single acoustooptic modulator shifting the reference beam by $40 \mathrm{MHz}$. It is used to measure the OPD introduced by the main delay line in SUSI. The metrology feeds PAVO with measurements of the displacement of the main delay line so that PAVO can track stellar fringes in a closed servo loop by driving the main delay line to compensate any fringe motion during observation. Since a detailed description of this metrology system in its original design was given by Davis et al. (1999a) and later by Robertson et al.
(2012) after the system underwent a recent upgrade, it will not be discussed further in this paper.

The remaining three metrology systems are newly installed and used for phase-referenced narrow-angle astrometric observations. Other than additional light sources, which consist partly of those shown in Fig. 4(b), the metrology systems do not require any extra hardware or optical components. The systems are made up of a whitelight (WL) metrology, a single laser (SL) metrology and a dual laser (DL) metrology system. Together, they form a two-prong approach to measuring the optical path length between fringe packets of two stars in a binary system. First, the WL and the SL metrology systems are used to measure the OPD of every optical path non-common to PAVO and MUSCA. The objective is to ensure that any change in the position of a MUSCA phase-referenced fringe packet is caused by the DDL within the beam combiner. Second, the DL metrology system is used to measure the displacement of the DDL.

A schematic diagram showing the optical paths of SUSI is given in Fig. 6. The labels in bold in the figure represent the different parts of SUSI. The labels in regular type font are optical components which can be cross-referenced back to Figs. 4 and 5. The non-common paths between PAVO and

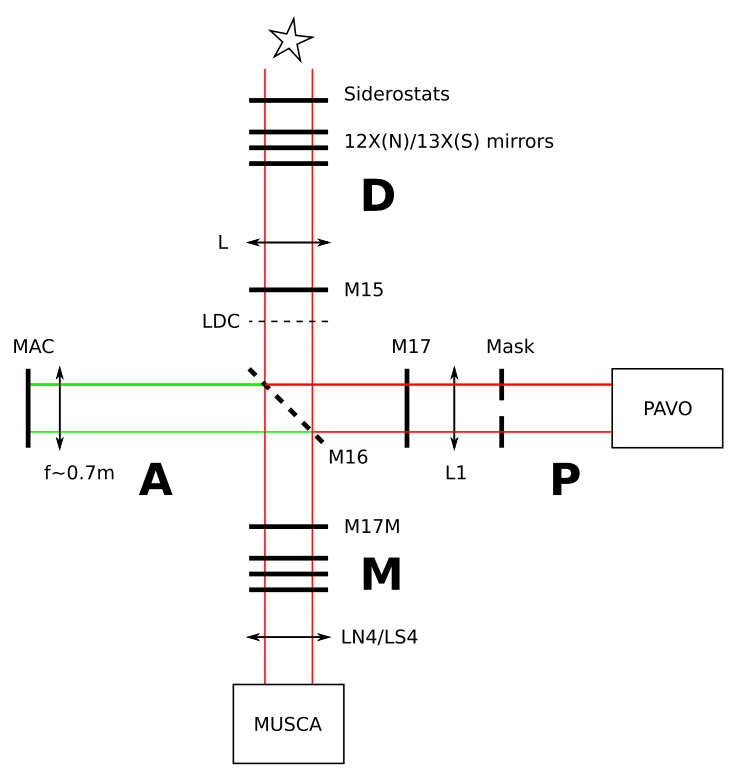

Fig. 6. Schematic of beam combiners at SUSI. Part D includes all the optical components from the siderostat until M16 (a collective term for N16 and S16). Part P consists of all optical components from M16 to the PAVO camera. Part A includes MAC (collective term for NAC and SAC), LS3 and LN3. Part M consists of all the optical components from M16 to the MUSCA APDs. 
MUSCA are labeled as $\mathbf{P}$ and $\mathbf{M}$ in Fig. 6. In the first prong of the approach, the phase delays of interference signals in MUSCA, $m$, are estimated using the phase delays of interference signals measured by PAVO, $z$, and the OPDs of the noncommon paths measured by the metrology systems. This is given as,

$$
\begin{aligned}
m & =\mathrm{OPD}_{\mathrm{D}}+\mathrm{OPD}_{\mathrm{M}} \\
& =z+\left(\mathrm{OPD}_{\mathrm{M}}-\mathrm{OPD}_{\mathrm{P}}\right),
\end{aligned}
$$

where,

$$
z=\mathrm{OPD}_{\mathrm{D}}+\mathrm{OPD}_{\mathrm{P}},
$$

and the subscripts in the equations represent different parts of the SUSI optical paths as illustrated in Fig. 6. Then in the second prong of the approach, the true phase delay difference between two MUSCA fringe packets, $S$, is estimated by correcting the apparent phase delay difference with the change in the OPD within MUSCA, $\triangle \mathrm{OPD}_{\mathrm{M}}$. This is given as,

$$
S=\left(m_{2}-m_{1}\right)+\Delta \mathrm{OPD}_{\mathrm{M}},
$$

where $m_{i}$ is the phase delay of either fringe packet.

In this paper, the phase delay of an interference signal is defined as the OPD between two arms of an interferometer computed at a fiducial location and is usually quoted in units of $\mu \mathrm{m}$. The fiducial location for MUSCA is in the middle of the scan range of the scanning mirror. Similarly, the fiducial location for PAVO is in the center of the FOV of the EMCCD camera. The fiducial locations for computing the remaining OPD terms in Eqs. (2) and (3) are not important because they are not measured independently.

\subsection{White-light metrology}

The light source for this metrology system is an LED which emits light at a peak wavelength of $\lambda_{\mathrm{I}}=\sigma_{\mathrm{I}}^{-1}=0.940 \mu \mathrm{m}$. It is injected into the SUSI optical path in front of PAVO with a pellicle beamsplitter (see Fig. 4(b)). It then propagates to a pair of retro-reflecting mirrors, NAC and SAC, and back to PAVO. This optical setup constitutes a Fizeau interferometer. Less than $5 \%$ of the total light from the LED is reflected into MUSCA because the dichroic mirrors (N16 and S16) are only highly reflective at wavelengths shorter than $0.79 \mu \mathrm{m}$. This has negligible effect on the phase-referencing performance of PAVO and MUSCA.
The optical path of the metrology is coaxially aligned with the incoming starlight path in order to minimize the non-common path error in the measurement. The interference fringes formed by the LED are recorded simultaneously with the stellar fringes during observation with the same PAVO camera for the same purpose. The lower quantum efficiency of the camera at the metrology wavelength $(\sim 25-30 \%)$ enables PAVO to observe fringes of a star and the metrology simultaneously. Suppose $w$ represents the phase delay of the fringes formed by this metrology, then $w$ is the sum of OPDs along the propagation path of the metrology, i.e.,

$$
w=2 \mathrm{OPD}_{\mathrm{P}}+2 \mathrm{OPD}_{\mathrm{A}} .
$$

However, the phase measurement is modulo of $2 \pi$. Therefore the non-ambiguity range (NAR) of the metrology is equal to one wavelength of the LED light. Despite the short NAR, the range is adequate because fringes are recorded by the PAVO camera at a rate of one frame per $\sim 5 \mathrm{~ms}$ and the OPD measured by $w$ is not expected to change by more than one wavelength within this time interval.

Over longer timescales, the range of OPD variation measured by $w$ can exceed one wavelength of light over a timescale of several hours depending on the laboratory seeing. This is not a concern because the PAVO spectrograph disperses the metrology fringes into narrow bands so that the coherence length of the fringe packet is $\sim 30 \mu \mathrm{m}$. The sum of $\mathrm{OPD}_{\mathrm{P}}$ and $\mathrm{OPD}_{\mathrm{A}}$ is not expected to vary beyond $30 \mu \mathrm{m}$ in one night as there are no moving parts along the optical path.

The WL metrology measures only the relative OPD. The reference OPD is taken to be the OPD at the start of an observation session. The value is near to zero (within $\pm 10 \mu \mathrm{m}$ approximately). The absolute value of the reference OPD is not important nor does it need to be precisely at zero because it will be canceled out in the data analysis.

The major source of noise in the phase delay measurement is photon noise because the LED is relatively bright as seen with the PAVO's low read noise EMCCD camera. The estimated measurement uncertainty is $80 \mathrm{~nm}$ or less, but this value does not translate directly into astrometric error because the error in determining the relative position of the phase-referenced fringe packet is averaged over many MUSCA scans, which reduces the error by a factor of $\sqrt{N_{\mathrm{SC}}}$, where $N_{\mathrm{SC}}$ is the number of good scans used in the integration. It does however affect 
the overall integration time because more scans are required to average out larger uncertainties.

\subsection{Single-laser metrology}

The light source for this metrology system is a green $\mathrm{He}-\mathrm{Ne}$ laser which emits light at a peak wavelength of $\lambda_{\mathrm{G}}=\sigma_{\mathrm{G}}^{-1}=0.5435161 \mu \mathrm{m}$. This laser also serves a dual purpose for optical alignment. The metrology laser is injected into the SUSI optical path the same way as the LED but it propagates via the pair of retro-reflecting NAC and SAC mirrors and into the MUSCA beam combiner. The intensity of the metrology laser is controlled by the selection of neutral density filters at the source (see Fig. 4(b)) so that it does not 'blind' the APDs when observing faint stars. This optical setup constitutes a MachZehnder interferometer.

Like the WL metrology, the optical path of the SL metrology is also coaxially aligned with the starlight path in order to minimize the non-common path error in the measurement. For the same reason, the interference fringes formed by the laser at the MUSCA beam-splitter are recorded by the MUSCA APDs simultaneously with the stellar fringes during observation. Suppose $x$ represents the phase delay of the fringes formed by this metrology, then the OPD probed by $x$ is,

$$
x=\mathrm{OPD}_{\mathrm{P}}+2 \mathrm{OPD}_{\mathrm{A}}+\mathrm{OPD}_{\mathrm{M}} .
$$

Although the phase measurement is modulo of $2 \pi$, the NAR is adequate because the scanning mirror makes an up and down scan (one period) per $150 \mathrm{~ms}$ and the OPD measured by $x$ is not expected to change by more than one laser wavelength within this time interval. Over longer timescales, when laboratory conditions are unstable, the OPD variation measured by $x$ can exceed one laser wavelength over a timescale of several hours, but is still negligible compared to the coherence length of the laser.

The SL metrology measures only the relative OPD between the two arms of the interferometer. The OPD measured at the start of an observation session is set as the reference OPD. It is also not necessary for the reference OPD to be precisely zero although it is usually set to near zero (within $\pm 5 \mu \mathrm{m}$ approximately) before the start of an observation. The absolute value of the reference OPD is not important because it will be canceled out in the data analysis.
Similar to the WL metrology, the measurement uncertainty of $x$ does not translate directly into astrometric error.

\subsection{Dual-laser metrology}

The light sources for this metrology system are green and red $\mathrm{He}-\mathrm{Ne}$ lasers. The former is the same green laser used for the SL metrology. The latter emits light at a peak wavelength of $\lambda_{\mathrm{R}}=$ $\sigma_{\mathrm{R}}^{-1}=0.6329915 \mu \mathrm{m}$. The intention of using two lasers of different wavelengths is to extend the NAR of a single laser metrology. Conventionally, this is achieved using a long synthetic optical wavelength by means of heterodyne interferometry (Daendliker et al., 1988; Schuhler et al., 2006) but the dual wavelength metrology in MUSCA employs only a simple homodyne fringe counting detection scheme, thereby avoiding the need for specialized optical elements and higher development cost. Neither laser is frequency stabilized, but based on the full width half maximum (FWHM) of their theoretical gain profiles the relative uncertainties of the laser wavelengths are better than $3 \times 10^{-7}$. This level of uncertainty is adequate because the displacement of the DDL is typically less than $0.5 \mathrm{~mm}$. Suppose $d$ represents the physical quantity measured by this metrology, then the OPD probed by $d$ is,

$$
d=\Delta \mathrm{OPD}_{\mathrm{M}},
$$

where $\triangle O P D_{M}$ is the change in optical path within MUSCA brought about by the displacement of the DDL. This metrology can achieve a measurement uncertainty of $\lesssim 5 \mathrm{~nm}$ and is discussed in detail in a separate paper (Kok et al., 2013a).

\section{Data Reduction Pipeline}

Raw data from a PAVO+MUSCA observation, in the form of interferograms, are reduced in two stages to produce its primary observable, which is the differential phase delay of two fringe packets. The first stage of the data reduction pipeline computes $m$ in Eq. (2) using estimates of $z, w$ and $x$ while the second stage computes $S$ in Eq. (4) by using estimates of $d$.

The PAVO interferograms are 32 by 512 pixels images recorded in $4.2 \mathrm{~ms}$ of exposure time. Figure 7 shows an example of a PAVO interferogram. They contain images of three pupils, one to be used for fringe tracking (left in the figure) and two to be used for tip-tilt correction (middle and right in the 


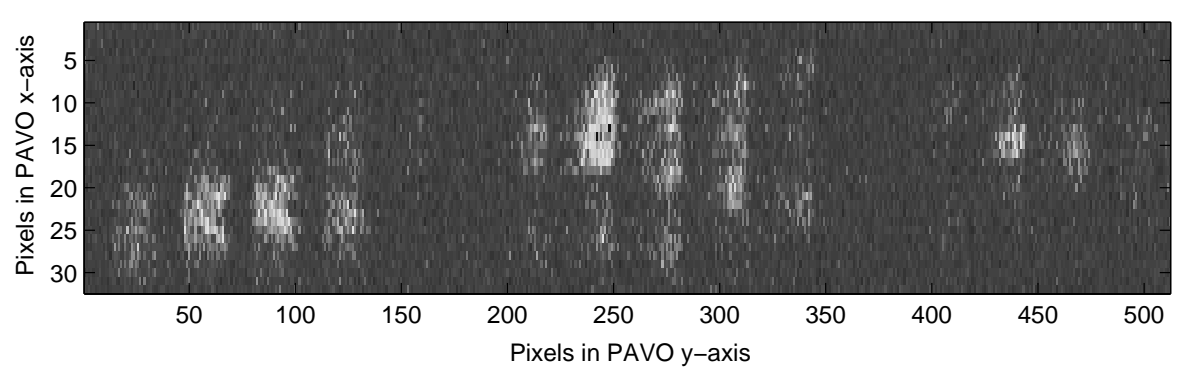

Fig. 7. An interferogram of $\alpha$ Gru recorded by PAVO. It contains images of spectrally dispersed (horizontally) pupils as sampled by an array of four lenslets. The science pupil is on the left of the frame. The pupils in the middle and right of the frame are used for tip-tilt control. They are ignored by the data reduction pipeline.

figure). Each pupil is imaged by 4 lenslets and spectrally dispersed by a prism. Each dispersed segment of a pupil is 33-pixel wide, which represents individual spectral channels of the PAVO spectrograph. The PAVO interferograms are stored as FITS files with headers that contain various information such as the status of the fringe lock, timestamp, etc.

The MUSCA interferograms are time series of photon counts recorded by the pair of APDs as the scanning mirror scans through a predetermined range periodically. The photon counts are stored as ASCII text files and each count has a timestamp for synchronization with post-processed data from PAVO in the pipeline. The temporal fringes recorded by the two APDs have a phase difference of $\pi$ rad due to the beam-splitter. Figure 8 shows several scans of MUSCA interferograms with the upper panel illustrating the out-of-phase relationship between signals from the two APDs. Let the interference signal of the time series be defined as the difference between the photon counts recorded by APD0 and APD1 and the photometry signal of the series be defined as the sum of the photon counts recorded by the APDs. The interference signal of the same scan in the upper panel of Fig. 8 is the lowest line in the lower panel in
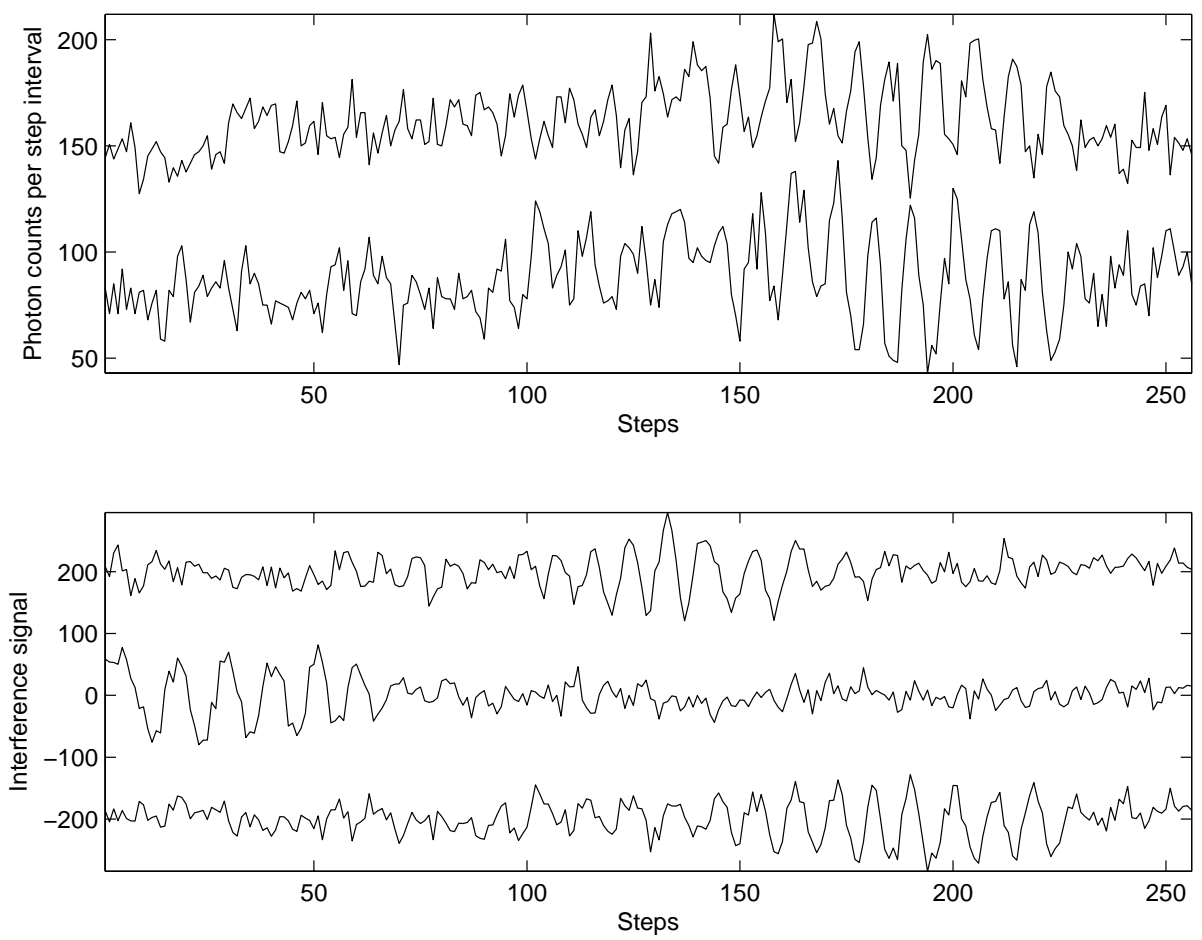

Fig. 8. Interferograms of $\beta$ Cru recorded by MUSCA. The top plots are photon counts recorded by the pair of APDs, one on each output port of the beam-splitter, hence the $\pi$ rad phase difference for the fringe signals. The bottom plots show the difference of photon counts recorded by the APDs. Three separate sweeps through the fringe envelope are depicted, illustrating the varying position of the fringes at different times. 
the same figure. Also plotted in the lower panel are interference signals of two other scans at different times which show the position of stellar fringe packets vary with time due to atmospheric turbulence.

\subsection{Stage I}

The main goal of the first stage of the pipeline is to produce a phase-referenced fringe packet that has an average phase error better than $5 \mathrm{~nm}$. This stage is implemented in two parts, one for each type of interferogram. The PAVO part of the pipeline is modified from an existing $V^{2}$ pipeline developed for the PAVO $V^{2}$ science observation (Ireland et al., 2013, in prep.). It is written in the IDL programming language. On the other hand, the MUSCA part of the pipeline was developed from scratch by the first author and is written in MATLAB. A diagram showing the interdependency between the PAVO and the MUSCA parts in this stage of the pipeline and the summary of their various subprocesses is given in Fig. 9. Only subprocesses within the shaded region in the figure are related to the phase-referencing part of the PAVO+MUSCA pipeline. The following subsections describe some of the more important subprocesses in the figure in the context of phasereferencing.

\subsubsection{Estimating $z$}

Only PAVO interferograms with stellar fringes are selected for further processing. First, the interferograms are pre-processed to retain only the relevant parts of the image (i.e. the spectrum of the science pupil) and to correct for a small distortion in the spectrum (along the $x$-axis of the image, the spatial fringes direction) which is caused by the glass prism. Then the spatially modulated fringes are rectified by treating the fringes in each spectral channel as an analytic signal and removing the carrier frequency through a series of forward and inverse Fourier transform operations and data manipulation in the Fourier domain. The outcome of the rectification is a complex function that describes the envelope of the fringes in each spectral channel, which is given as,

$$
\rho_{j, k}=\Omega_{j, k} e^{-i \phi_{j, k}},
$$

where $j$ and $k$ are indices of a pixel in the $y$ - and $x$-axis respectively (see Fig. 7 ) while $\Omega_{j, k}$ is the function of the fringe envelope and $\phi_{j, k}$ is a phase term attributed to the OPD of the combining beams. The index $j$ refers to one of the 33 spectral channels while $k$ refers to one of the 4 lenslets in PAVO. Only the first 21 channels are within the PAVO science bandwidth and used for estimating $z$. Suppose $\sigma_{j}$ represents the wavenumber of a spectral channel and $z\left(\sigma_{j}\right)$ or $z_{j}$ is the OPD between two arms of SUSI at a particular wavenumber, then, according to Tango (1990),

$$
\sigma_{j} z_{j}=\bar{\sigma} z(\bar{\sigma})+\xi\left(\sigma_{j}-\bar{\sigma}\right)+\psi_{j},
$$

where $\bar{\sigma}$ is the mean of the wavenumbers of all the spectral channels, $\xi$ is the group delay of the fringes at $\bar{\sigma}$ and $\psi_{j}$ represents the higher order terms of the Taylor's expansion of $\sigma_{j} z_{j}$. With an additional noise term, $\varepsilon_{j, k}$, to represent atmospheric phase

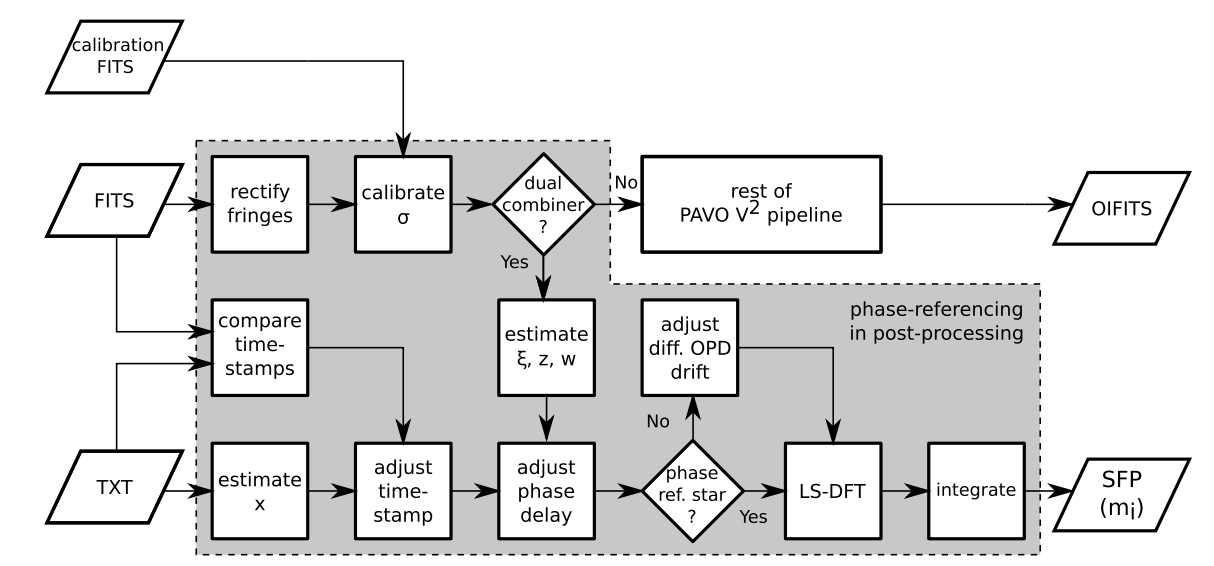

Fig. 9. The logical flow of Stage I of the PAVO+MUSCA pipeline. Subprocesses within the shaded region are the phasereferencing part of the pipeline. The main inputs to the pipeline are the PAVO (FITS) and MUSCA (TXT) interferograms. If necessary, calibration FITS files which contain PAVO interferograms of metrology light sources are also included to calibrate the PAVO spectrograph. The output is a phase-referenced fringe packet (SFP). 
noise and/or optical aberration, then the argument of $\rho_{j, k}$ in Eq. (8) is,

$$
\phi_{j, k}=2 \pi \sigma_{j} z_{j}+\varepsilon_{j, k} .
$$

The first step in estimating $z_{j}$ is to estimate the group delay of the fringes. This is done as follows. First, the autocorrelation of the phasor $p_{j}$ is calculated. The phasor, $p_{j}$, is defined as,

$$
p_{j}=\sum_{k} \rho_{j, k}=\bar{\Omega}_{j} e^{-i \bar{\phi}_{j}}=\bar{\Omega}_{j} e^{-i\left(2 \pi \sigma_{j} z_{j}+\bar{\varepsilon}_{j}\right)},
$$

and its autocorrelation is,

$$
\begin{aligned}
q_{a} & =\sum_{j} p_{j} p_{j+a}^{*} \\
& =e^{-i 2 \pi a \Delta \sigma \xi} \sum_{j} \bar{\Omega}_{j} \bar{\Omega}_{j+a} e^{-i 2 \pi \Delta \psi_{j, a}},
\end{aligned}
$$

where $a \Delta \sigma=\sigma_{j}-\sigma_{j+a}$ and $\Delta \psi_{j, a}=\psi_{j}+\bar{\varepsilon}_{j}-$ $\left(\psi_{j, a}+\bar{\varepsilon}_{j+a}\right)$. The wavenumber difference between two adjacent spectral channels, $\Delta \sigma$, is sufficiently regular across the entire spectral bandwidth to be considered constant and to have negligible effect on the following analysis step.

Next, the Fourier transform of the vector $\mathbf{q}$ from Eq. (12) is calculated using a Fast Fourier Transform (FFT) routine. Using the notation $\hat{\mathbf{q}}$ to denote the Fourier transform of $\mathbf{q}$, then,

$$
\hat{q}_{b}=\delta_{b-\check{b}} * \hat{\bar{\Omega}}_{b}=\hat{\bar{\Omega}}_{b-\check{b}},
$$

where $\delta_{b-\check{b}}$ is a delta function at index $\check{b}$ and $\breve{b}=\Delta \sigma \xi N_{\mathrm{FFT}}$. The second term of the convolution in Eq. (13) is the power spectral density (PSD) of $\bar{\Omega}_{j} e^{-i\left(\psi_{j}+\bar{\varepsilon}_{j}\right)}$ and has a global maximum at the origin. As a result the group delay of the fringes can be estimated from the position of the global maximum of $\hat{q}_{b}$. The estimated group delay is,

$$
\tilde{\xi}=\Delta \sigma^{-1} \tilde{b} / N_{\mathrm{FFT}},
$$

where $\tilde{b}$ is the location of the global maximum of $\hat{q}_{b}$, which may not necessarily be at $\breve{b}$ as discussed in the next paragraph, and $N_{\mathrm{FFT}}$ is the size of (or number of elements in) the vector as input to the FFT routine. The tilde notation in Eq. (14) and throughout this paper denotes estimation of a parameter. Since the coherence length of each spectral channel, $\Delta \sigma^{-1}$, is $\sim 30 \mu \mathrm{m}$, this estimate is reliable if the true group delay is within $\pm 15 \mu \mathrm{m}$.

The assumption that $\psi_{j}$ is approximately constant across the bandwidth of the PAVO spectrograph is ensured by the usage of a longitudinal dispersion compensation system, or LDC (Davis et al., 1999a). However the similar assumption regarding $\bar{\varepsilon}_{j}$ is only true when the atmospheric phase noise across the pupil is small (i.e. seeing is good). If the phase noise variation is large then the location of the global maximum of $\hat{q}_{b}$ may not be at zero. In addition, a large variation also gives rise to spurious peaks in the PSD.

In order to gauge the phase noise variation, a signal-to-noise (SNR) ratio, $\eta$, is introduced. $\eta$ is defined as the ratio of the value of the dominant peak to the noise floor of the PSD where the noise floor is defined as the standard deviation of the PSD with the dominant peak removed. The value of $\eta$, which is inversely proportional to the phase noise variation, is also used to gauge the accuracy of the OPD estimate, $\tilde{z}_{j}$, because the requirement is that $\tilde{\xi}$ must be accurate to within one wavelength. From computer simulation, an $\eta$ of 5 is acceptable while an $\eta$ of 6 is good (Kok et al., 2013b).

If the optical media in which the starlight propagates to reach the beam combiners are nondispersive then the group delay is also the OPD affecting the fringes at all wavenumbers. However, except for the optical path in the vacuum pipe near the siderostats, all optical media along the optical path of PAVO and MUSCA are dispersive.

The last step in estimating $z_{j}$ is to adjust the group delay estimate by a fraction of a wavelength according to the dispersion factor, $\delta_{\ell}$, and length of the optical path propagated by the light source, $L_{\ell}$. Suppose $\alpha$ is the value to be adjusted and its estimate is defined as,

$$
\begin{aligned}
\alpha & =\sum_{\ell=1}^{N_{\mathrm{MED}}} \delta_{\ell} L_{\ell}, \quad \text { or }, \\
e^{i \tilde{\alpha}} & =\left\langle e^{i \bar{\phi}_{j}} e^{-i 2 \pi \sigma_{j} \tilde{\xi}}\right\rangle,
\end{aligned}
$$

where $N_{\text {MED }}$ is the number of different optical media along the propagation path, then,

$$
\tilde{z}_{j}=\tilde{\xi}+\frac{\tilde{\alpha}}{2 \pi \sigma_{j}} .
$$

Finally, suppose $\sigma_{\mathrm{M}}$ is the mean wavenumber of the MUSCA's operating bandwidth, which is $\sim 1.2 \mu \mathrm{m}^{-1}$, then the OPD estimate at that wavelength is obtained by evaluating Eq. (16) at $\sigma_{\mathrm{M}}$.

\subsubsection{Estimating $w$}

The WL metrology fringes are recorded at the same time as the stellar fringes in PAVO. After undergoing the same pre-processing, the metrology 
fringes are used to estimate $w$ in a slightly different way than the stellar fringes to estimate $z$. Since the optical paths probed by $w$ are contained within an enclosed climate controlled environment, they are not expected to vary beyond one wavelength of the metrology fringes within the one unit of a PAVO interferogram exposure time. Therefore the OPD probed by the metrology can be measured by converting the phase of the metrology fringes, which is the argument of $p_{j}$ in Eq. (11), into distance. The estimate of $w$ is given as,

$$
\tilde{w}=\frac{2 \pi N_{\sigma \mathrm{I}}+\arg \left(p_{\mathrm{I}}\right)}{2 \pi \sigma_{\mathrm{I}}}
$$

where the subscript $I$ is the spectral channel at $\sigma_{\mathrm{I}}$ and $N_{\sigma \mathrm{I}}$ represents an integer number of cycles over which the fringes may have wrapped around. $N_{\sigma \mathrm{I}}$ is obtained by unwrapping the phase measurement collected throughout several sets of observations (or the entire night) because the phase measurement obtained directly from a 'single' wavelength fringes is modulo of $2 \pi$. The top plot in Fig. 10 shows the argument of $p_{\text {I }}$ without taking into account phase wrapping.

\subsubsection{Estimating $x$}

The SL metrology fringes are also recorded by MUSCA together with science fringes. As a result of that, the interference signal of the metrology fringes imposes a measurement grid across the scan range of the scanning mirror. The spacing of the lines,
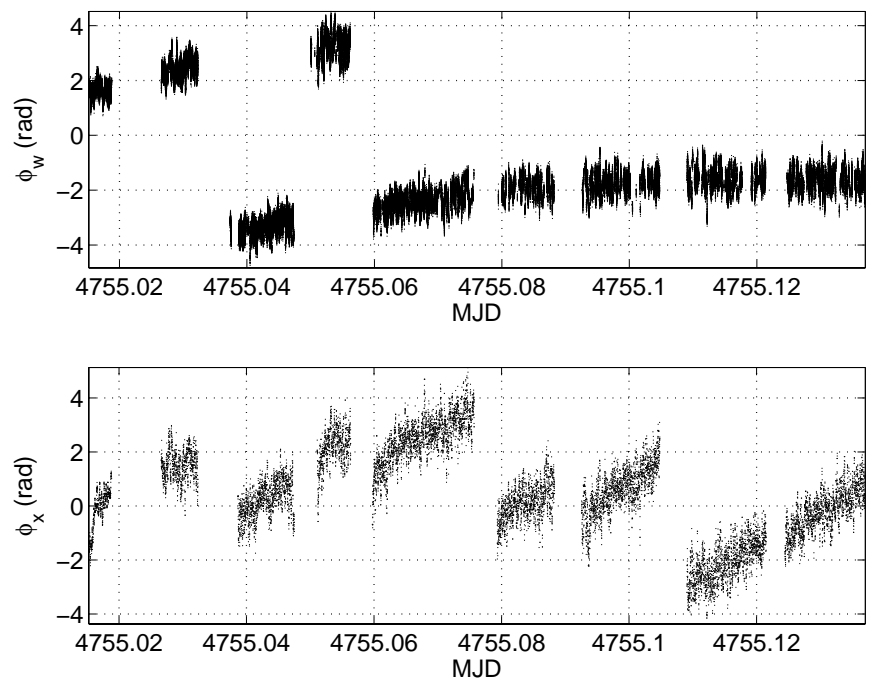

Fig. 10. Wrapped phases of the WL (top) and the SL (bottom) metrology fringes used to estimate $w$ and $x$ respectively.

which is defined by the wavelength of the metrology laser, is used to measure the change in OPD induced by the mirror. A visualization of this grid is shown in Fig. 11(a). Instead of using a Fourier filtering method, the faint metrology fringes are extracted by shifting a pre-recorded scan of high SNR reference fringes in the spatial domain and the amount of shift to be applied is measured from the phase of the metrology fringes recorded during the observation. This method assumes that the metrology fringes produced by each scan of the scanning mirror in $\mathrm{OPD}$, denoted by $\ell(x)$, is a spatially shifted version

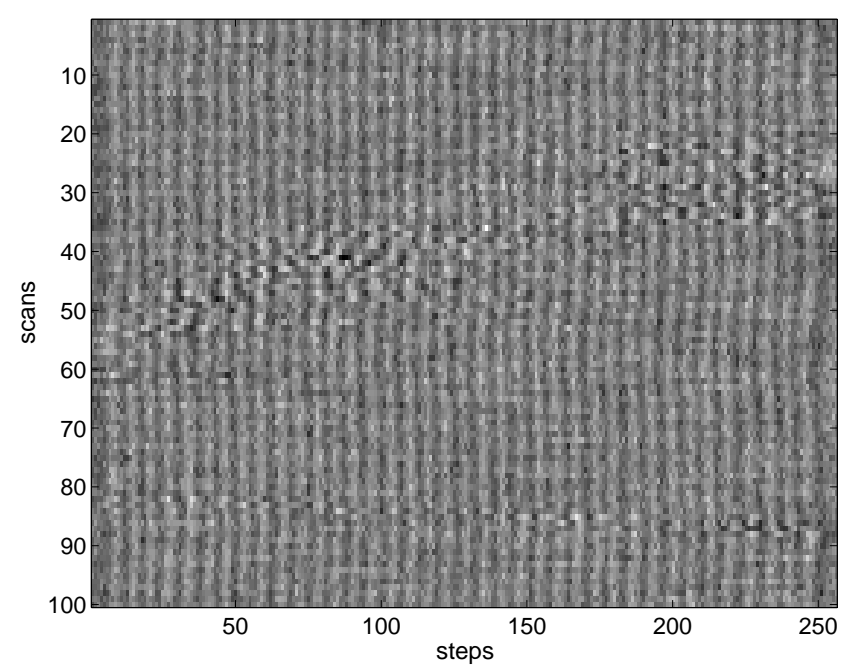

(a)

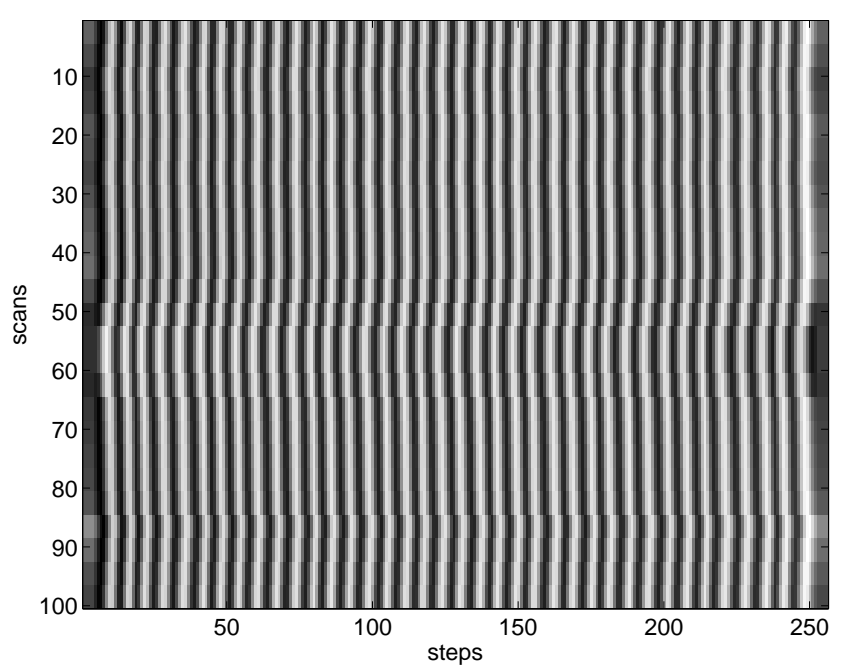

(b)

Fig. 11. Waterfall plot of (a) SL metrology and MUSCA science fringes as recorded and (b) SL metrology fringes after SNR enhancement and removal of the science fringes. 
of fringes produced by a reference scan, $\ell_{\mathrm{R}}(x)$. Physically, the spatial shift is caused by internal laboratory seeing which has a timescale ${ }^{(a)}$ of several tens of seconds to a minute. This is very slow compared to the time taken by the scanning mirror to complete an up and down scan cycle, which is about $150 \mathrm{~ms}$. Therefore the assumption that path length variations are much less than one wavelength between successive scans is reasonable. Suppose $\hat{\ell}_{\mathrm{R}}$ represents the Fourier transform of $\ell_{\mathrm{R}}(x)$ and the $\tilde{\ell}(x)$ represents an estimate of $\ell(x)$, then,

$$
\tilde{\ell}(x)=\mathcal{F}^{-1}\left\{\hat{\ell}_{\mathrm{R}} \exp \left(i \frac{\sigma}{\sigma_{\mathrm{G}}} \phi_{\mathrm{G}}\right)\right\},
$$

where $\phi_{\mathrm{G}}$ is phase of the metrology fringes recorded during observation. A waterfall plot of a set of recovered metrology fringes (or $\tilde{\ell}$ ) over time is shown in Fig. 11(b). The phase variation of the metrology fringes in Fig. 11(a) is preserved in Fig. 11(b). However, the SNR of the fringes is enhanced significantly.

The phase delay of the fringes is then computed by inverting the function, $\tilde{\ell}(x)$. Mathematically,

$$
\tilde{x}=\frac{N_{\sigma \mathrm{G}}}{\sigma_{\mathrm{G}}}+\tilde{\ell}^{-1}(\tilde{\ell}(x)) .
$$

The function $\tilde{\ell}(x)$, which is in the form of,

$$
\tilde{\ell}(x)=A \cos \left(2 \pi \sigma_{\mathrm{G}} x+\phi_{\mathrm{G}}\right) \text {, }
$$

is not invertible by definition due to the periodic nature of a cosine function but with additional computation (e.g. the derivative of the function), $\tilde{x}$ can be recovered for the entire scan range.

The phase delay in the middle of the scan range is defined to be zero thereby providing a reference point for measuring positions of phasereferenced fringe packets in the next stage of the pipeline. However, like the measurement with the WL metrology, the phase measurement of the SL metrology fringes must also be corrected for phasewrapping. The term $N_{\sigma \mathrm{G}}$ in Eq. (19) is added to represent an integer number of cycles that the fringe phase has wrapped around and is obtained by unwrapping the phase measurement collected throughout several sets of observations. The lower plot of Fig. 10 shows the phase of the SL metrology fringes before unwrapping.

\footnotetext{
${ }^{\mathrm{a}}$ Duration over which the average phase variation is $1 \mathrm{rad}$, computed from the spectral density of the measured phase variation.
}

\subsubsection{Estimating $m$}

The phase delay of the MUSCA fringes can be estimated by combining phase delay measurements from PAVO and the two metrology systems discussed in Secs. 3.1 and 3.2, which is given as,

$$
\tilde{m}=\tilde{z}-\tilde{w}+\tilde{x} .
$$

The values of $\tilde{z}$ and $\tilde{w}$ are linearly interpolated to simulate the same sampling rate as $\tilde{x}$ before Eq. (21) is computed. In order to avoid confusion, the values of $\tilde{m}$ do not represent the photon counts in the MUSCA interferograms. Instead, they represent $\mathrm{OPD}$ at each step in one mirror scan. If the stellar fringes are to be plotted as a graph, the photon counts are on the ordinate and values of $\tilde{m}$ are on the abscissa.

\subsubsection{Coherent integration}

In order to reduce the uncertainty in the phase delay estimation of $\tilde{m}$, a number of scans can be coherently integrated together. This means the photon counts are summed up according to their OPDs. However, only the photon counts with reliable estimates of $m$ (has $\tilde{z}$ of $\eta \geq 5$ ) are integrated. Due to contribution from the other phase delays, $\tilde{m}$ does not have regular sampling. Therefore, it is easier to integrate the fringes in the Fourier domain by first computing the Fourier transform of the fringes using a discrete Fourier transform (DFT) algorithm which is capable of computing the Fourier transform of a non-uniformly sampled signal (Scargle, 1989). The computed Fourier transforms have regular frequency spacing in the Fourier domain. The algorithm also allows the frequency spacing in the Fourier domain to be appropriately specified. Furthermore, by integrating the fringes in the Fourier domain, additional phase error due to interpolation in the time domain can be avoided. Figures 12 and 13 show the result of coherently integrated phasereferenced fringe packets. They are also the output of this first stage of the pipeline.

The phase-referenced fringe packet in Fig. 12 is a result of observing a target in self phasereferencing mode. This means both PAVO and MUSCA were observing the same star. The phasereferenced fringe packet in Fig. 13 is a result of observing a target in dual-star phase-referencing mode. In this mode, PAVO observed one component of a binary star while MUSCA observed the other. Due to their relative position in the sky, the separation between fringe packets changes with time as 

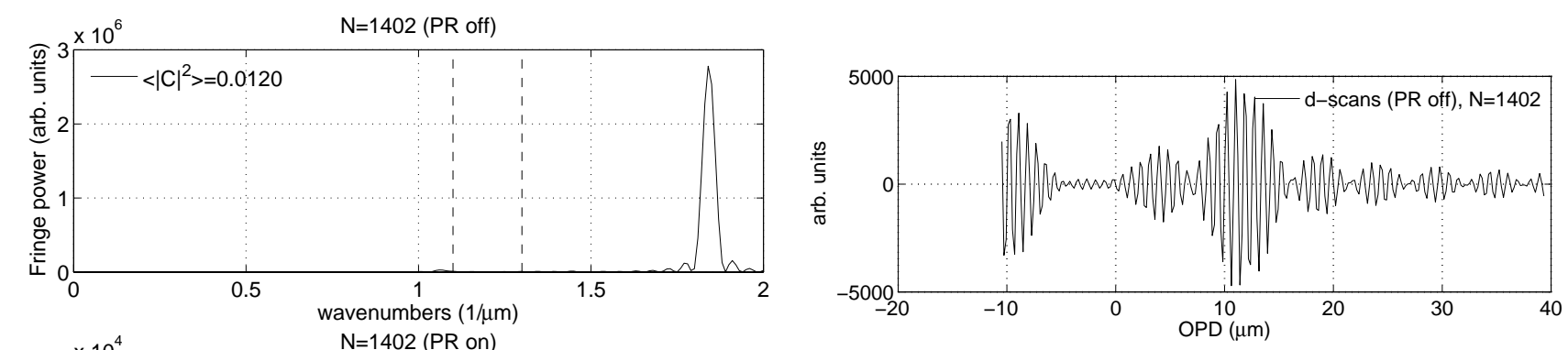

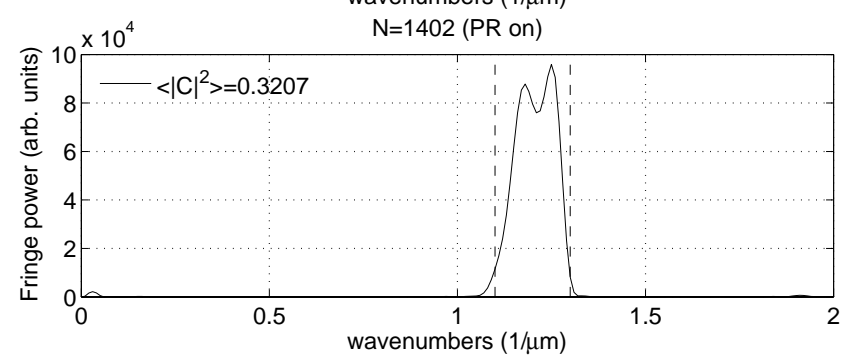

(a)

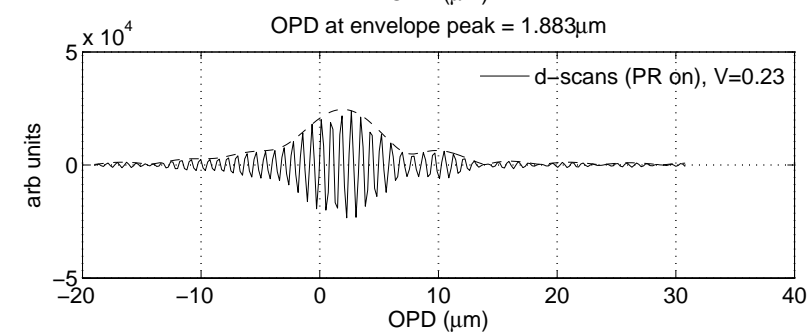

(b)

Fig. 12. Plots in (a) show the incoherently (top) and coherently (bottom) integrated Fourier transform of a self phasereferenced fringe packet of $\delta$ Orionis Aa. The peak in the top plot of (a) belongs to the SL metrology laser because the metrology fringes are coherently integrated without phase-referencing. However, when phase-referencing is engaged the fringes are incoherently integrated and hence the absence of the metrology laser peak in the bottom plot. The signal within the two vertical dashed lines in the bottom plot of (a) belongs to the star. Plots in (b) show the inverse Fourier transforms of (a) but only with signal between the dashed lines.

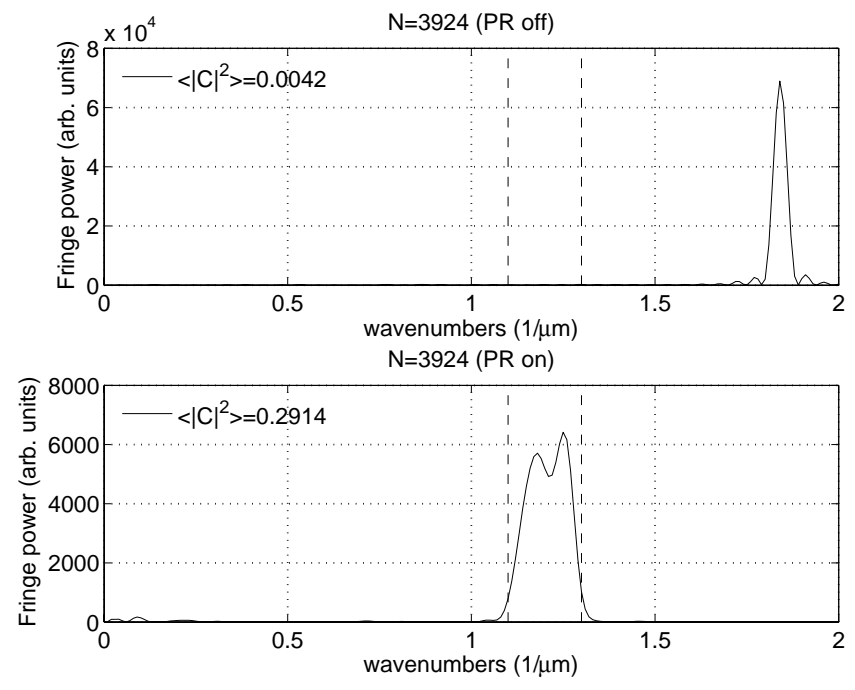

(a)
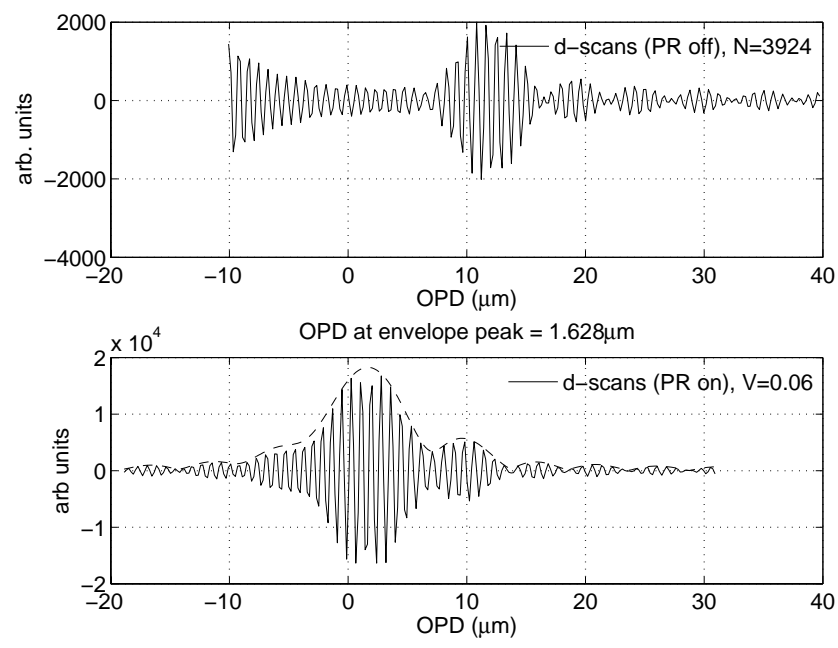

(b)

Fig. 13. Similar to Fig. 12. Plots in (a) show the incoherently (top) and coherently (bottom) integrated Fourier transform of a dual phase-referenced fringe packet of $\delta$ Orionis $\mathrm{Ab}$, which is separated by $\sim 0.3^{\prime \prime}$ from the reference star $\delta$ Orionis Aa. Plots in (b) show the inverse Fourier transforms of (a) but only with signal between the dashed lines.

the pair of stars traverse the night sky. Therefore a correction term, $\Delta z$, must be added to Eq. (21) in order to accurately estimate the phase delay of the MUSCA fringes, otherwise the integration will yield fringes of very low or zero visibility because they are incoherently summed. Eq. (21) for dual-star phase-referencing becomes,

$$
\tilde{m}=(\tilde{z}+\Delta z)-\tilde{w}+\tilde{x} .
$$

In its simplest form, $\Delta z$ is a function of drift velocity and elapsed time (with respect to a chosen reference time, usually chosen to be at the start of the dual-star observation). However, the pipeline 
uses an astronomical model, which is based on the position angle and the on-sky separation of two stars, to compute $\Delta z$. The same model is also used for narrow-angle astrometry and is discussed in Sec. 5. Optimizing the amplitude of the coherently integrated fringes by tweaking the position angle parameter in the model does not immediately yield the astrometry of the two stars because $\Delta z$ is also affected by the group delay drift effect, which is also discussed in Sec. 5.

\subsubsection{Phase error}

The coherence of fringes across multiple scans can be assessed using a metric which is defined as,

$$
\left\langle|C|^{2}\right\rangle=\frac{\int_{0}^{\infty}|\langle\hat{I}(\sigma)\rangle|^{2} d \sigma}{\int_{0}^{\infty}\left\langle|\hat{I}(\sigma)|^{2}\right\rangle d \sigma},
$$

where $\hat{I}$ is the Fourier transform of the phasereferenced fringes and the notation \langle\rangle denotes an average over multiple scans. The metric measures the weighted average of the phase variation across the Fourier transform of the fringes. Similar to the Strehl ratio for measuring the performance of an adaptive optics system, this metric has a value between 0 and 1 . If the fringe integration is perfectly coherent over all scans, then the metric will have a value of 1 . On the other hand, if the fringes are completely out of phase then the metric will have a value of 0 . Now, if the phase variation is random and has a normal distribution, then,

$$
\left\langle|C|^{2}\right\rangle \approx e^{-\sigma_{\varphi}^{2} / 2}
$$

where $\sigma_{\varphi}$ in this context is the standard deviation of the phase variation. The average phase variation can then be estimated from the coherence metric.

\subsection{Stage II}

The main goal of the second stage of the pipeline is to measure the separation of two fringe packets at high precision. This stage is executed when the previous stage has produced two or more phasereferenced fringe packets. In this stage, the apparent and true separation of a pair of phase-referenced fringe packets are computed. Figure 14 illustrates the logical flow of this stage of the pipeline, which is written entirely in MATLAB.

\subsubsection{Estimating d}

The dual-laser metrology is used to measure a change in the position of the DDL in MUSCA.

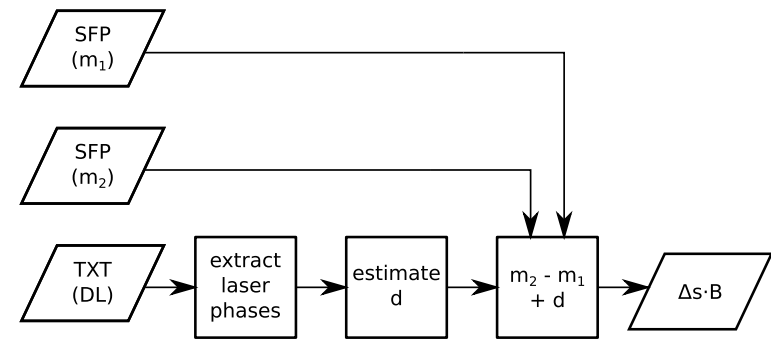

Fig. 14. The logical flow of Stage II of the PAVO+MUSCA pipeline. The main inputs comprise the output of Stage I and a set of MUSCA interferograms containing only the DL metrology fringes. The output is the true separation of the given pair of fringe packets.

The DDL is static when stellar fringes are being recorded and it is only moved to alternate between different fringe packets. Therefore, $\tilde{d}$ is a one-off measurement of the DDL displacement between successive observations for which metrology fringes are recorded just before and after the DDL is moved. The estimated change in optical path is given as,

$$
\tilde{d}=\frac{\left(\Delta \varphi_{2}-\Delta \varphi_{1}\right) / 2 \pi+\Delta N}{\sigma_{\mathrm{R}}-\sigma_{\mathrm{G}}},
$$

where the numerator is the difference between the phases of the red and green laser fringes at two different delay line positions indicated by the subscript 1 and 2 , while $\sigma_{\mathrm{R}}$ and $\sigma_{\mathrm{G}}$ in the denominator are the wavenumbers of the two lasers in vacuum. The details of data reduction for this metrology are discussed in a separate paper (Kok et al., 2013a). Note that the term $\tilde{d}$ in Eq. (25) is equal to $n_{\mathrm{M}} \mathrm{d}$, where $n_{\mathrm{M}}$ is the refractive index of air at the mean MUSCA wavenumber $\sigma_{\mathrm{M}}$ and $\mathrm{d}$ is the displacement of the DDL defined in Eq. (2) in (Kok et al., 2013a).

\subsubsection{Estimating the true separation, $S$}

The apparent separation of the two fringe packets, $\Delta m$, is estimated from the phases of the product of the Fourier transform of one fringe packet and the conjugate of the Fourier transform of the other fringe packet. Examples of the modulus amplitude and phase of the Fourier transform products are shown in the middle and lower plots of Fig. 15. The phase delay at the mean wavenumber is a measurement of the apparent separation of the fringe packets, $\Delta \tilde{m}$, and is estimated from a linear least squares fit. Examples of the goodness of fit, $\chi^{2}$, of possible values of $\Delta m$ are also plotted in Fig. 15 . The value that has the global minimum $\chi^{2}$ is usually 

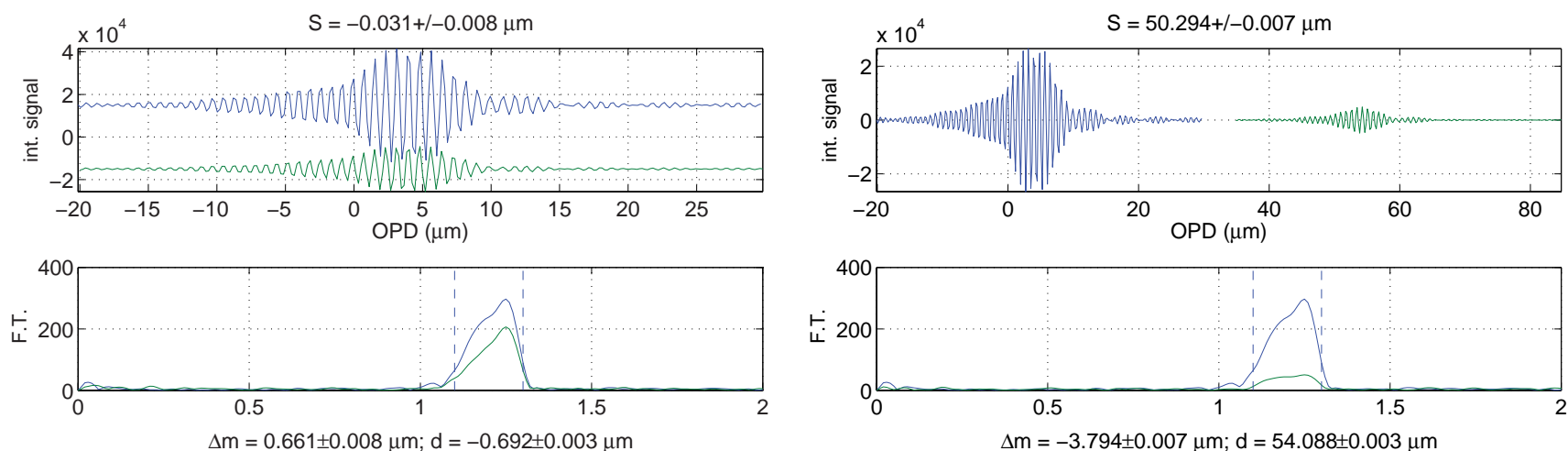

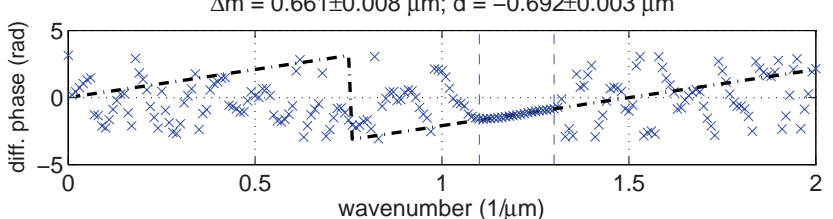

(a)

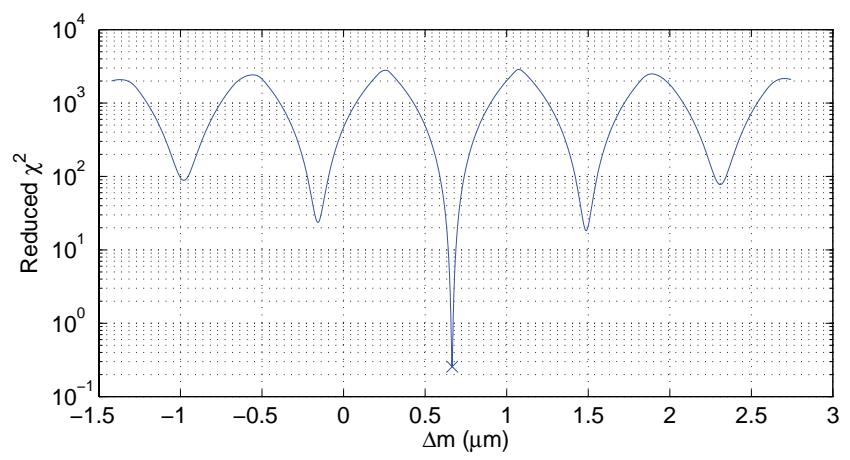

(c)

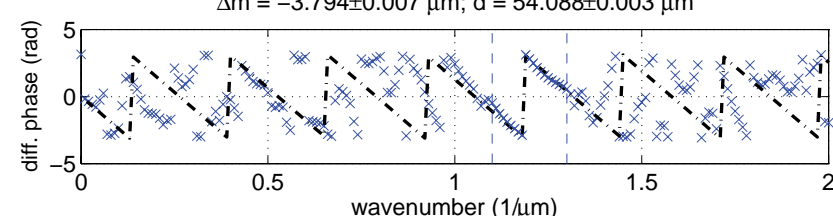

(b)

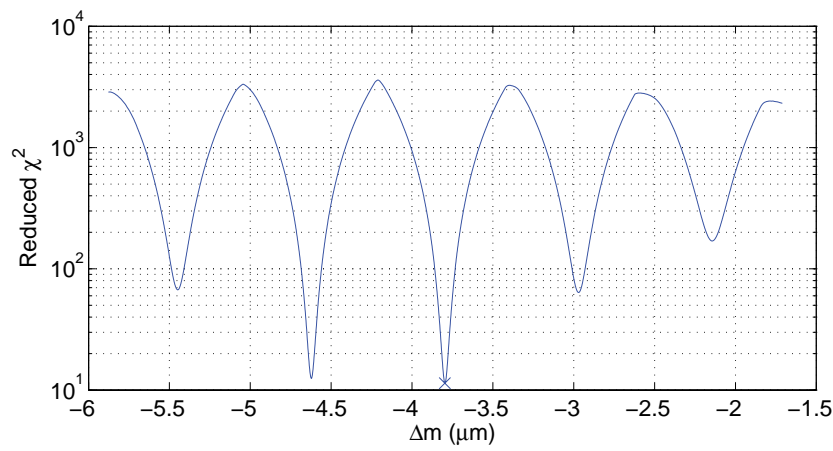

(d)

Fig. 15. The top plot in (a) shows the phase-referenced fringe packet of $\delta$ Orionis Aa observed at two different times while (b) shows the fringe packet separation of $\delta$ Orionis Aa and Ab. The level of the interference signals in the top plot of (a) has been arbitrarily adjusted for clarity. The middle plots show the modulus amplitude of the Fourier transform of each fringe packet in the top plot. The bottom plots show the phase difference of the Fourier transform of the fringe packets (middle plots). The middle and bottom plots have the same $x$-axis scale. The portion of the phase signal that determines the apparent separation of the fringe packets, $\Delta m$, lies between the two vertical dashed lines. The dash-dotted lines show the least-squares fit to the phases in that region. The plots in (c) and (d) are the goodness of fit, $\chi^{2}$, of possible values of $\Delta m$ to the phases in (a) and (b) respectively. It is usually but not always the case that the value having the global minimum $\chi^{2}$ (indicated by the symbol $\times$ ) is associated with the accepted value for $\Delta m$.

but not always the case accepted as the value for $\Delta \tilde{m}$. The presence of severe differential dispersion (between two fringe packets) and an unresolved fringe packet embedded within one of the two fringe packets used in the analysis may cause the result of the fit to be ambiguous by one wavelength. Example of cases due to the latter are discussed in Sec. 6 . The true separation of a fringe packet pair, $S$, is then given as,

$$
\tilde{S}=\Delta \tilde{m}+\tilde{d}
$$

where $\Delta \tilde{m}=\tilde{m}_{2}-\tilde{m}_{1}$ is the estimated apparent separation of the fringe packets. The true separations of the fringe packets in the examples in Fig. 15 are indicated in the top most plots.

\section{Narrow-Angle Astrometry}

A complete set of MUSCA astrometric observations consists of a series of fringe packet acquisitions. Fringes of a reference (primary component of a binary) star, then a target (secondary component of a binary) star and finally back to the reference star are acquired in sequential order. This is done so that the systematic error in phase delay of the fringe packet caused by the group and phase delay drift can be corrected. 


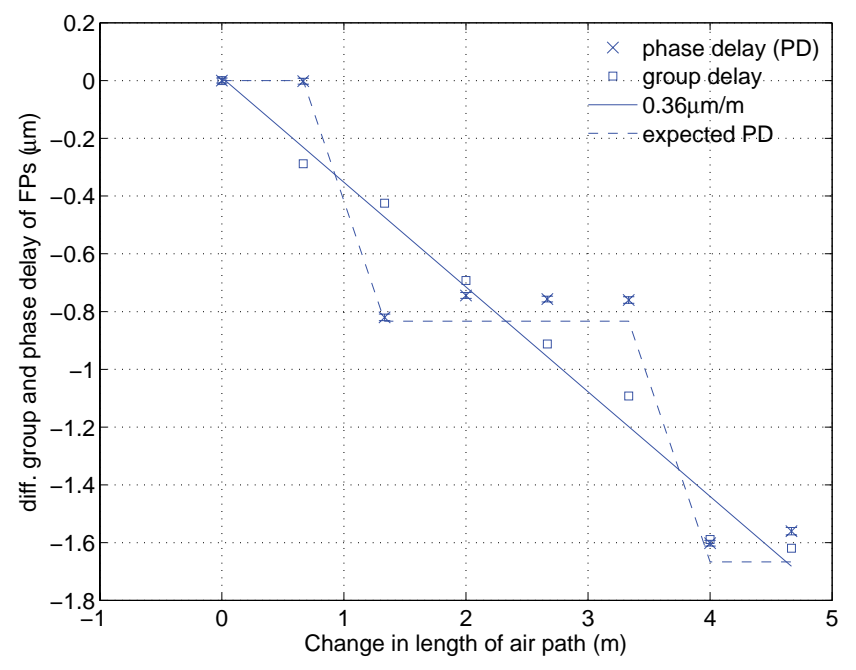

(a)

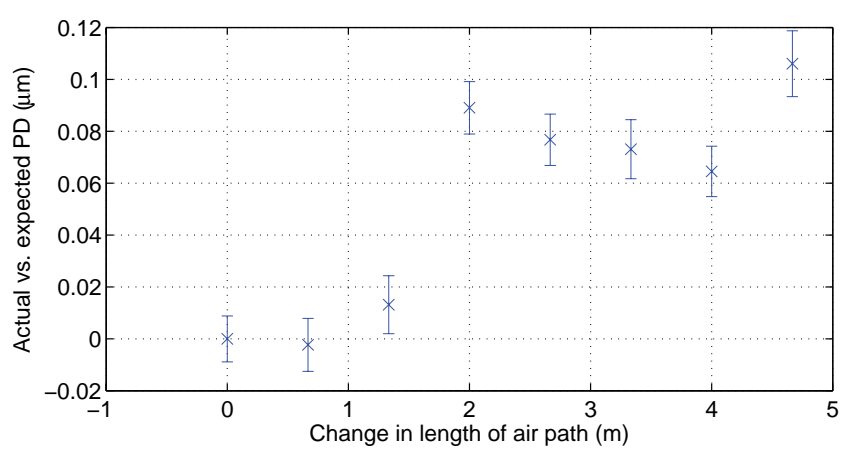

(b)

Fig. 16. (a) Relative position (group and phase delay) of a fringe packet (FP) showing the effect of group delay drift. (b) The actual versus the expected phase delay $(\mathrm{PD})$ of the FP showing the effect of phase delay drift. Both plots are derived from the PAVO and MUSCA simulators without atmospheric phase noise.

\subsection{Group delay drift}

The group delay drift occurs because optical media used to equalize the optical path in SUSI (air and glass) are dispersive and the mean operating wavelengths of PAVO and MUSCA are not the same. The rate of the drift is proportional to the length of the optical media along the common optical path of the interfering starlight and the difference of group delays at PAVO and MUSCA operating wavelength is about $0.36 \mu \mathrm{m}$ per $1 \mathrm{~m}$ of air path. This estimate, which used extrapolated numbers from Tango (1990), takes into account the proportionality of optical path length in both air and glass used in SUSI. Figure 16 plots the differential group and phase delay (at mean wavenumber) of a fringe packet as extracted from the phases of its Fourier transform. The computation is similar to the one discussed in the previous section for computing $\Delta \tilde{m}$ but the reference fringe packet in this case is a fringe packet from the same source but constructed with a different amount of air path. Essentially the group delay in the plot shows the relative position of a fringe packet of source when the amount of air path is varied. The fringe packet in the figure is simulated with the PAVO and MUSCA simulator. Although the group delay drifts according to the linear relation with the air path length, the differential phase delay of the fringe packet varies in discrete steps of about one MUSCA wavelength. This is expected because the measurement is phase sensitive and does not change unless the differential group delay changes by more than half a MUSCA wavelength from its previous value.

Due to this group delay drift effect, the separation between the primary and the secondary fringe packets must be corrected to yield a value that depends only on the projected separation of the binary star. Suppose $\xi_{S, P}$ represents the differential group delay between the primary and the secondary fringe packets which is interpolated from the slope of the phases of the Fourier transform of the cross-correlation of the two primary fringe packets (e.g. the lower plots of Figs. 15(a) and 15(b)) of a given astrometric observation, then the amount of adjustment to the fringe packet separation is,

$$
\Delta \tilde{S}=\xi_{S, P}+\frac{1}{2 \pi \sigma_{\mathrm{M}}} \arg \left(e^{-i 2 \pi \sigma_{\mathrm{M}} \xi_{S, P}+i \phi_{S, P}}\right),
$$

where $\phi_{S, P}$ is a correction to the phase of the fringe packet due to a similar phase delay drift effect which is discussed in the next section. In most cases, $\Delta \tilde{S}$ is small $\left(\phi_{S, P} / 2 \pi \sigma_{\mathrm{M}}\right)$ because the amount of air path change within the time span of one bracketed astrometric observation is usually shorter than $3 \mathrm{~m}$ and therefore $\xi_{S, P}$ is usually less than half of one MUSCA wavelength.

\subsection{Phase delay drift}

The phase delay of the MUSCA fringes also drifts systematically in proportion to the length of the optical media along the common optical path of PAVO and MUSCA. This phenomenon occurs 
because the effect of higher order dispersion is not taken into account when estimating the phase delay at the mean MUSCA wavelength. The amount of systematic error is given as $\psi\left(\sigma_{\mathrm{M}}-\bar{\sigma}\right)$ where the function $\psi(\sigma-\bar{\sigma})$ was first introduced in Eq. (9) and is explained in detail by Tango (1990). The rate of drift is very small but can become significant when the change in the uncompensated dispersive media path length is large $(\sim 5-10 \mathrm{~m})$ or the longitudinal dispersion is not well compensated. A calculation based on refractive indices and dispersion coefficients extrapolated from Tango's (1990) to MUSCA's wavelength suggests that the air and glass combination in SUSI gives rise to phase delay drift of about $0.035 \mu \mathrm{m}$ for every $1 \mathrm{~m}$ air path of change. Figure 16(b) shows the effect of the phase delay drifts of a simulated fringe packet. Since the difference in air path is unlikely to change by more than $10 \mathrm{~m}$ within a time interval need to make a bracketed astrometric observation, this error is unlikely to cause an additional phase delay jump as seen in Fig. 16(a). The amount of phase delay adjustment, $\phi_{S, P}$, to be applied to the fringe separation is empirically interpolated from the measured phase delay difference between two primary fringe packets within a bracketed observation.

\subsection{Calibration}

Single unresolved stars are good targets for instrument calibration. They are used to test the stability of the reference phase of the fringes in MUSCA and consequently the intrinsic precision of the instrument and its data reduction pipeline. The intrinsic precision of the data reduction pipeline was evaluated with simulated data (Kok et al., 2013b) and the results plotted to illustrate group and phase delay shift are shown in previous sections. The reference fringe phase in MUSCA can change due to instrumental and astrophysical reasons. The main instrumental cause is a change in the position of the differential delay line (DDL) in MUSCA while the main astrophysical cause is a change in the photo center of the star under observation. Single unresolved stars are suitable because they appear as point sources and therefore do not exhibit any photo center shift.

Calibration tests were carried out in two stages. In the first stage, an internal white light source was used. The goal of the test is to assess the ability of the DDL to slew away and then slew back to (or close to) its original position as well as the ability of the dual-laser (DL) metrology to measure the displacement of the DDL. The reason an internal white light source is used at this stage is to eliminate any astrophysical effect and to isolate the cause of phase shifts to just the DDL and the metrology. The white light source is the tungsten bulb which is also used for internal fringe searching. The optical setup for this test is similar to the setup for the DL metrology (a Mach-Zehnder interferometer). The non-common paths between the white light source and the DL metrology are negligible because these two sources both originate from the same pin hole and are injected into the optical path by the same optical components (see Fig. 4(b)).

An example of the fringe packets of the internal white light source recorded after every subsequent DDL slew away and back are shown in the top panel of Fig. 17(a). The middle and bottom panels of the figure respectively show the modulus amplitude and phase of the Fourier transform of the crosscorrelation of the two fringe packets as described in Sec. 4.2.2. The differences in OPD or the separations (symbol $S$ ) between pairs of fringe packets (measured in $\mu \mathrm{m}$ ) from several consecutive iterations of this test are shown in Fig. 17(b). The first fringe packet is used as the reference and therefore is plotted with a fringe packet separation of zero. Similar but slightly different measurements, the phase differences between pairs of fringe packets (measured in radians) are listed in Table 1. This measurement is not applicable to the first data point in Fig. 17(b), hence the table has one data point less than the figure. The values in the table are equivalent to $2 \pi$ times the $1 / \sigma_{\mathrm{M}}$ modulo of the points in the plot in Fig. 17(b). $\sigma_{\mathrm{M}}$ is the mean wavenumber of the fringes which in this case, $\sigma_{\mathrm{M}} \approx 1.25 \mu \mathrm{m}^{-1}$ but for the actual stellar fringes $\sigma_{\mathrm{M}}=1.2 \mu \mathrm{m}^{-1}$.

The values in Table 1 provide a very useful metric to assess how well two fringe packets are aligned in terms of their phases. Ideally, a perfect alignment gives a zero phase difference while a less than perfect alignment gives a non-zero phase difference between $-\pi$ and $\pi$. A large phase difference indicates the presence of a large systematic error in the instrument which has a direct effect on its narrow-angle astrometric precision. Since the optical path of the interferometer was not changed during this test, the phase misalignment observed here is not due to the phase delay effect mentioned in Sec. 5.2. The range of phase differences measured with the internal white light source is between 0.1 


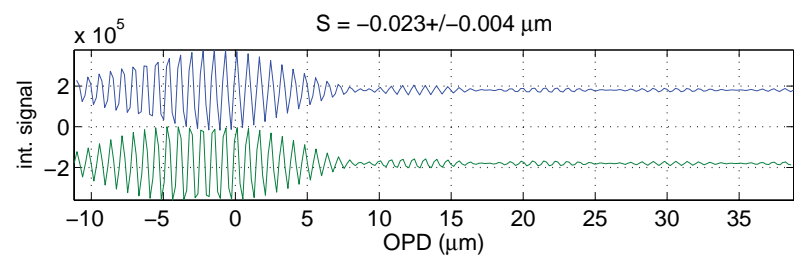

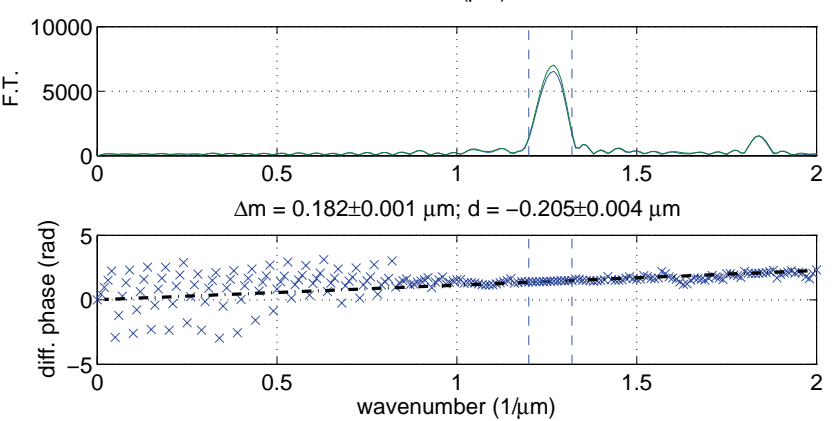

(a)

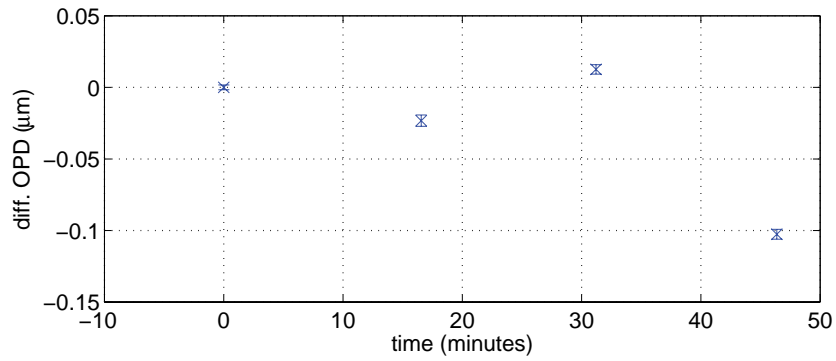

(b)

Fig. 17. Comparison between the phases of a pair of fringe packets. In (a), the top panel shows the two fringe packets which have their mean level adjusted so that their phase alignment can be clearly seen while the middle and bottom panels show the modulus amplitude and the differential phase of the Fourier transform of the two fringe packets respectively. The displacement of the DDL in MUSCA as measured by the DL metrology is indicated above the bottom panel. In (b), the fringe separations of several pairs of fringe packets are shown. The light source of the fringe packets in this figure is the internal laboratory tungsten bulb.

Table 1. Phase stability of the fringes of calibrators in MUSCA.

\begin{tabular}{lcr}
\hline $\begin{array}{c}\text { Light } \\
\text { source }\end{array}$ & $\begin{array}{c}\text { Related } \\
\text { figures }\end{array}$ & $\begin{array}{r}\text { Differential } \\
\text { phase (rad) }\end{array}$ \\
\hline \multirow{2}{*}{ Internal WL } & Fig. 17(b) ${ }^{\dagger}$ & $-0.18 \pm 0.03$ \\
& & $0.10 \pm 0.03$ \\
Achernar & Fig. 18(a) & $-0.81 \pm 0.03$ \\
& & $-0.02 \pm 0.10$ \\
& & $0.01 \pm 0.10$ \\
& & $0.02 \pm 0.09$ \\
& Fig. 20(a)-(b) & $-0.07 \pm 0.10$ \\
& & $-1.44 \pm 0.06$ \\
Bellatrix & Fig. 18(b) & $0.50 \pm 0.06$ \\
& & $-0.18 \pm 0.08$ \\
& Fig. 18(c) & $-0.08 \pm 0.08$ \\
& & $0.04 \pm 0.12$ \\
& Fig. 20(c) & $0.17 \pm 0.13$ \\
& Fig. 18(d) & $-0.69 \pm 0.16$ \\
& & $-0.10 \pm 0.11$ \\
& & $-0.07 \pm 0.10$ \\
\hline
\end{tabular}

${ }^{\dagger}$ with DDL displacement.

and $0.8 \mathrm{rad}$ or $\sim 10-100 \mathrm{~nm}$. The lower end of this range is good and is the level of precision required for high precision astrometry. However, the upper end of this range is poor.
In the second stage, a known unresolved single star (for a given baseline) was used. The goal in this stage is to assess the ability of the instrument as a whole (all SUSI subsystems, PAVO and MUSCA) to maintain phase stability during a dual-star phasereferencing observation. The optical setup is not a Mach-Zehnder interferometer as in the first stage but the actual MUSCA setup described in Sec. 2.2 for astrometric observation. Several known single stars have been used as calibrators but only a selected few are listed in Table 2 together with records on nights they are observed. These selected observations either have long enough observation time or were observed more than once in one night which allows their phase stability to be analyzed.

Similar to Fig. 17(b), the plots in Fig. 18 show the phase sensitive relative position of fringe packets (with respect to the first data point) of Achernar, Bellatrix, $\beta$ Lupi and $\phi$ Sagittarii. The fringe packets shown were recorded at different times but on the same nights for each star. The DDL in MUSCA was not moved during these observations. The results here show the intrinsic precision of the instrument and the data reduction pipeline without the effect of the DDL and the DL metrology. Fringe packets of Bellatrix and $\phi$ Sagittarii recorded at different times are plotted in Fig. 19 as examples. The results are discussed at the end of this section. 
Phase-Referenced Interferometry and Narrow-Angle Astrometry with SUSI

Table 2. Successful observations of calibrators.

\begin{tabular}{rlcccc}
\hline HR & Star & Baseline $^{\dagger}$ & Dates $^{\ddagger}$ & $\begin{array}{c}\text { \# of MUSCA } \\
\text { scans }^{\text {d }}\end{array}$ & $\begin{array}{c}\text { Integration } \\
\text { time }^{\text {d }}(\text { min })\end{array}$ \\
\hline 472 & Achernar & N3-S2 & 121120,121122 & $\begin{array}{c}1100-7000 \\
\sim 500\end{array}$ & $2-15$ \\
1790 & Bellatrix & N4-S2 & 130107 & $\sim 1$ \\
5571 & $\beta$ Lupi & N3-S3 & 130717,130720 & $200-400$ & $0.4-0.9$ \\
7039 & $\phi$ Sagittarii & N4-S2 & 130726 & $\sim 300$ & $\sim 0.6$ \\
\hline
\end{tabular}

${ }^{\dagger} \mathrm{N} 3-\mathrm{S} 2=20 \mathrm{~m}, \mathrm{~N} 3-\mathrm{S} 3=40 \mathrm{~m}, \mathrm{~N} 4-\mathrm{S} 2=60 \mathrm{~m}$.

${ }^{\ddagger}$ In YYMMDD format.

${ }^{\mathrm{d}}$ For one data point.

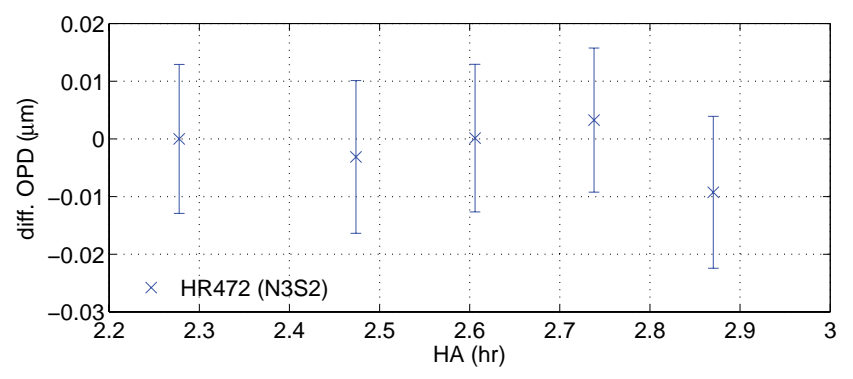

(a)

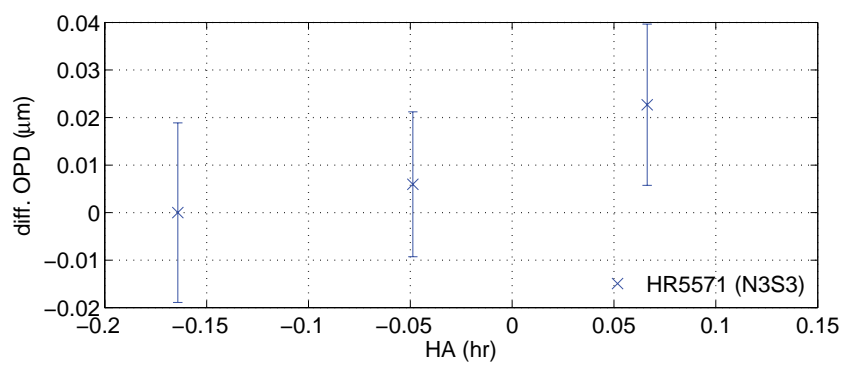

(c)

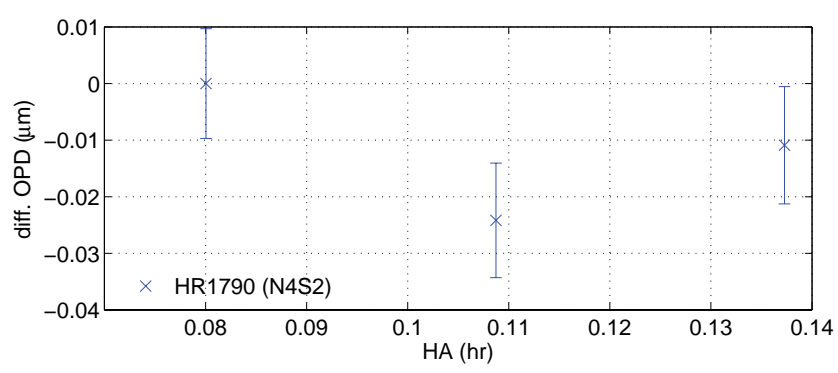

(b)

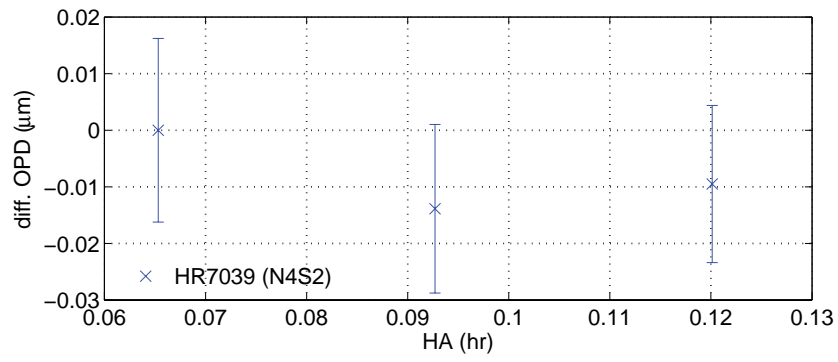

(d)

Fig. 18. Similar to Fig. 17(b) but single stars (indicated in the legend of each plot) replaced the tungsten bulb as light sources. No DDL displacement took place.
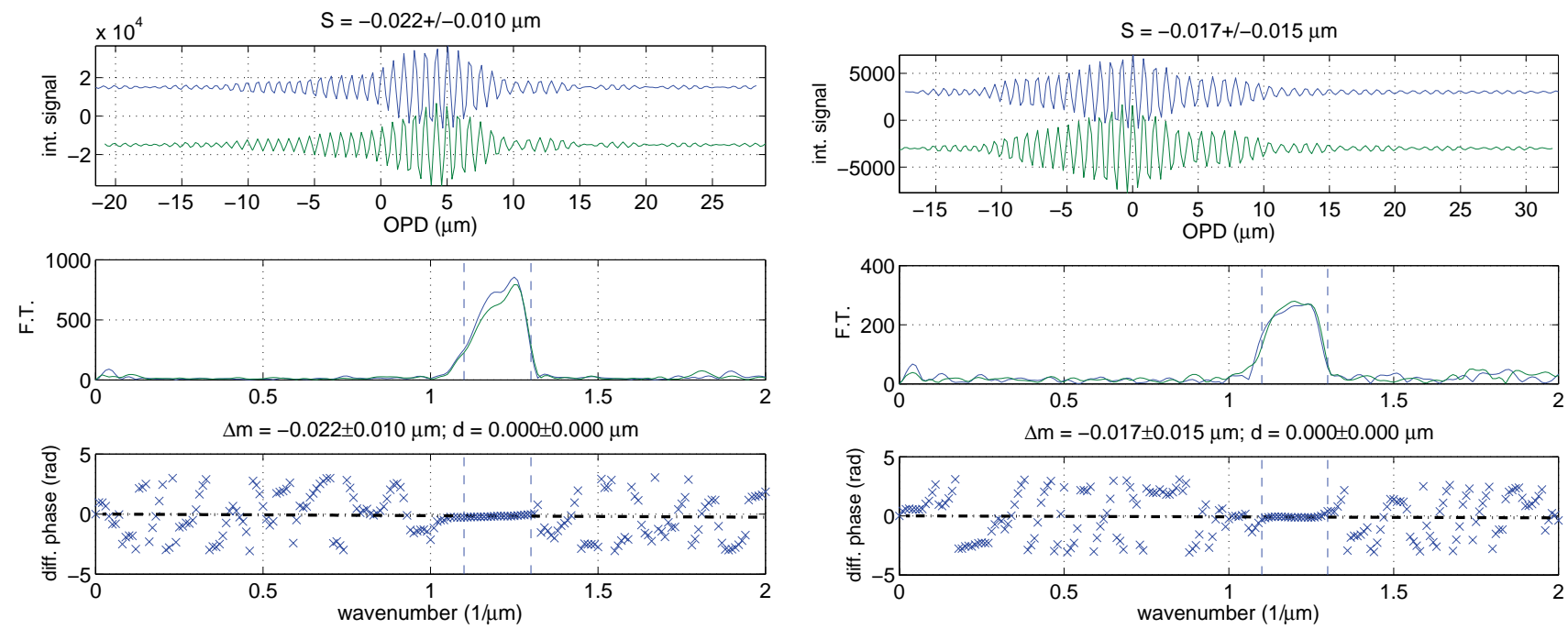

(a) Bellatrix

(b) $\phi$ Sagittarii

Fig. 19. Similar to Fig. 17(a) but single stars replaced the tungsten bulb as light sources. No DDL displacement took place. 

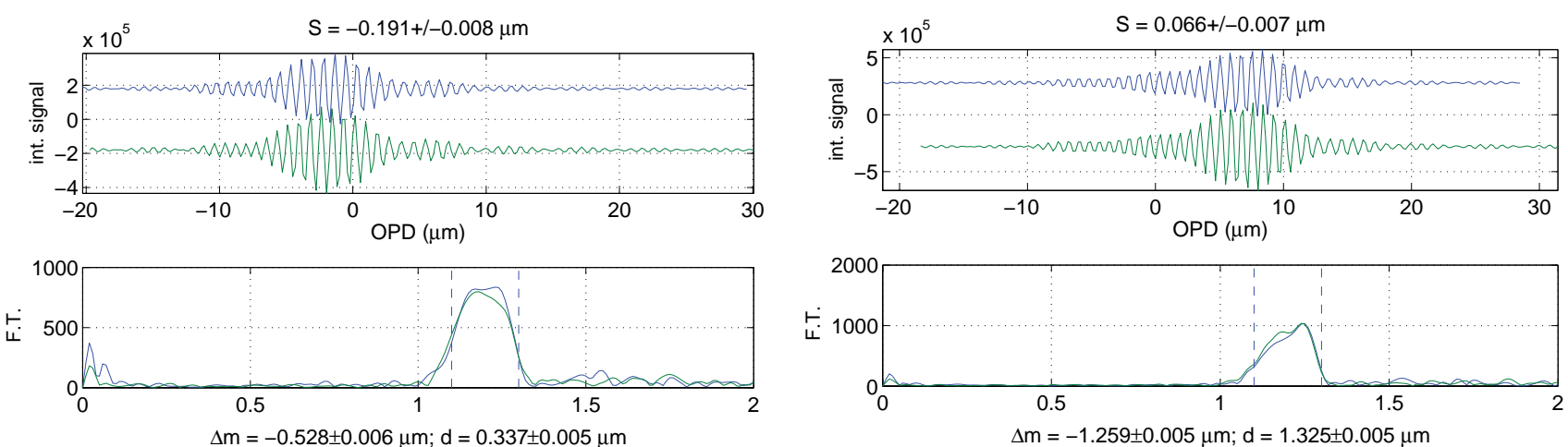

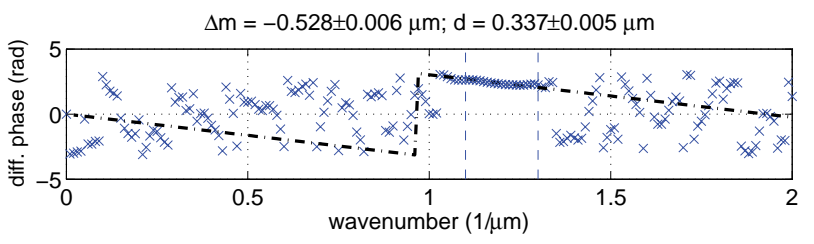

(a) Achernar

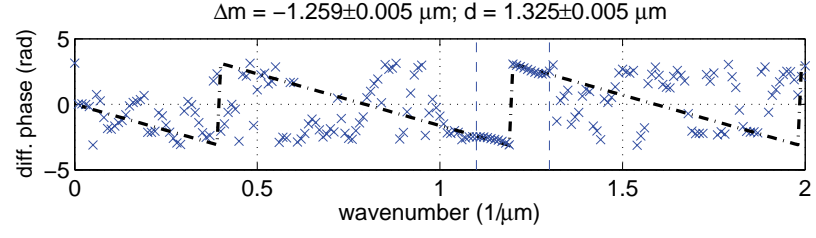

(b) Achernar
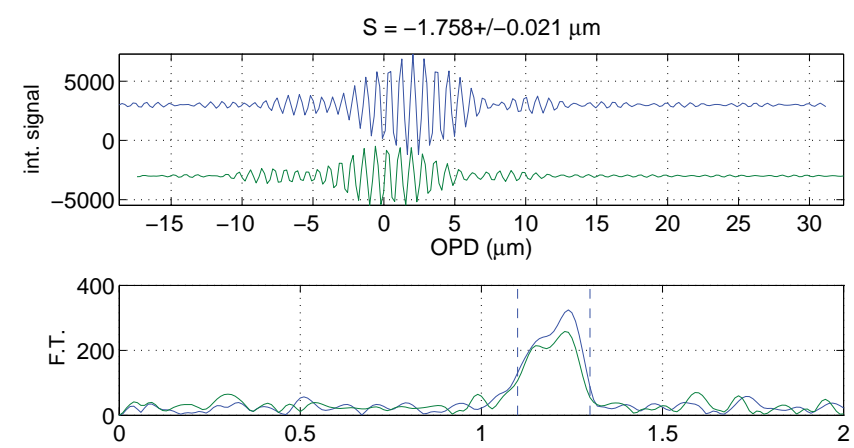

$\Delta \mathrm{m}=-1.467 \pm 0.020 \mu \mathrm{m} ; \mathrm{d}=-0.291 \pm 0.004 \mu \mathrm{m}$

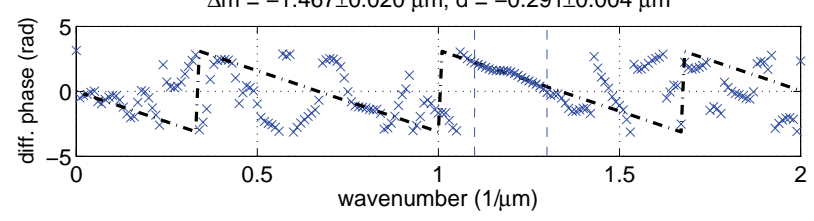

(c) $\beta$ Lupi

Fig. 20. Similar to Fig. 17(a) but single stars replaced the tungsten bulb as light sources. DDL in MUSCA was displaced after each fringe packet has been recorded. The amount of displacement as measured by the DL metrology is indicated above the bottom panel of each sub-figure.

With the DDL in MUSCA moved away and back after one fringe packet is recorded, the separations of pairs of fringe packets are shown in Fig. 20. The phase differences of the fringe packets are listed in Table 1. The phase misalignments reported in the table are due to the intrinsic misalignment observed in the previous section as well as the phase delay drift effect because the stellar fringe packets were observed at different times and hence with different air path length. The fringe separation of the pair of $\beta$ Lupi fringe packets in Fig. 20(c) is more than one MUSCA wavelength because of a change in the amount of air path in the main delay line as fringe packets were observed at different times in the night and the longitudinal dispersion compensator (LDC) was adjusted in between observations. Despite that, the phase alignment between the fringe packets is still less than $1 / 8$ th of the mean MUSCA wavelength $(<0.8 \mathrm{rad})$.

The results from the series of calibration tests discussed in this section indicate that the intrinsic precision of the instrument and the data reduction pipeline is better than $\sim 20 \mathrm{~nm}$ when the DDL in MUSCA is not displaced between observations but 
degrades to $\sim 100 \mathrm{~nm}$ when the DDL is displaced. This lower precision is not adequate for mircoarcsecond precision astrometry.

The correlation between the precision of measurement and the displacement of the DDL suggests that the accuracy of the DL metrology could be a problem despite having a precision of $\sim 5 \mathrm{~nm}$ or better. However further investigation revealed that the suggestion is not definitive. Firstly, the non-common path error arising from the difference in optical path between the metrology lasers and the white light source (tungsten bulb) is negligible because both light sources originate from the same pin hole and their fringes are recorded with the same photo-detectors. Secondly, the position of the linear stage on which the DDL is mounted varies due to changes in internal (electronics) and external temperature but this variation is measured by the SL metrology system and corrected for in all the tests. The low duty cycle $(<5 \%)$ motion pattern of the DDL (the ratio between the time spent slewing the stage to idle) during the above tests and observations ensured no excessive heat is generated by the motor driving currents in the electronics. Lastly, there were no obvious changes in the structure of the metrology laser pupils that could have altered the phase of the laser fringes.
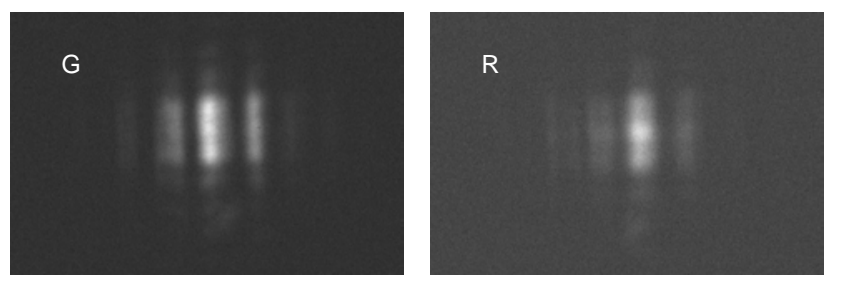

(a) $\mathrm{N}$ pupils (before)
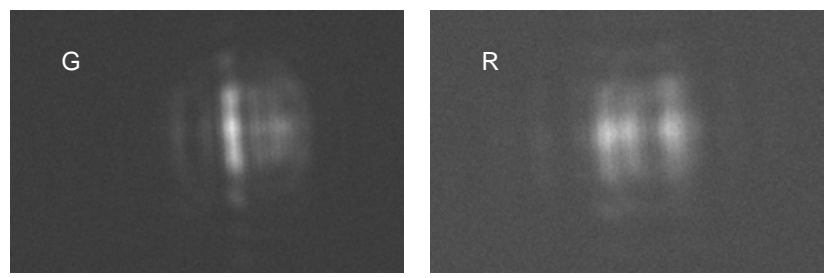

(c) S pupils (before)
Images of the laser pupils as seen with the MUSCA alignment CCD camera are shown in Fig. 21. The structures in the pupils originate from spurious reflections off the back side of the M16 dichroic filters, which are fabricated on 6-mm thick parallel glass windows (wedge angle of $\lesssim 30^{\prime \prime}$ ). The pupils of the green and red lasers are not identical and are labeled in the figure as "G" and "R" respectively. Neither are the pupils of the North and South beams identical. The exact cause of the observed asymmetries is uncertain but are probably due to the fabrication process. These structures are neither observed with the internal WL source, the IR LEDs at the siderostats nor starlight. The structures within the laser pupils are not expected to change because the spurious reflections occur before the laser beams enter into MUSCA (see Fig. 4(a) and 5(a)) and Fig. 21 clearly shows this. Due to the presence of such structures, the probability of unsuspectingly altering the phase of the laser fringes is not zero. The spatially modulated fringes across the laser pupils are sensitive to the differential tilt of the original and the displaced laser beam. Changes in the fringe pattern can alter the total intensity recorded by the MUSCA APDs at a given scan step and eventually the phase of the metrology fringes.
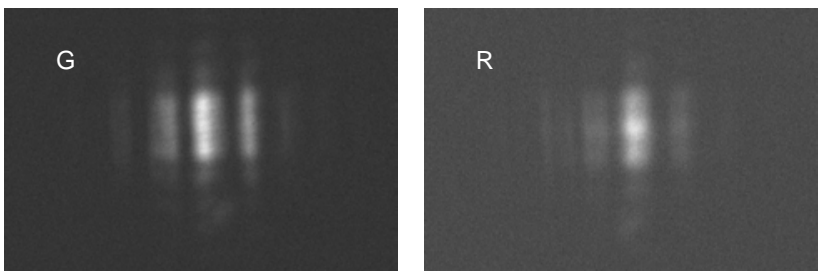

(b) $\mathrm{N}$ pupils (after)
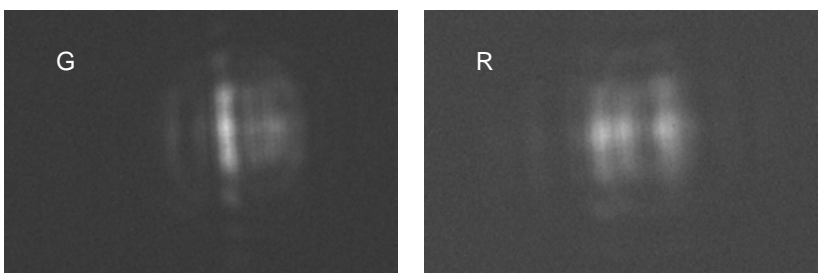

(d) S pupils (after)

Fig. 21. Images of the green $(G)$ and red $(R)$ laser pupils as seen with the MUSCA alignment CCD camera. The spatially modulated fringes like structures seen across the pupils are caused by overlapped and displaced pupils from spurious reflections off the back side of the M16 dichroic filters. The structures are not identical between the North (N) and the South (S) pupils because the 6 - $\mathrm{mm}$ parallel glass windows the dichroic filters were fabricated on most probably have different reflectivity profile (exact cause was not investigated). More importantly the structure within the pupils remains unchanged after the DDL is displaced and then slew back to its original position. This result is expected because the spurious reflections occur before the laser beams propagate into MUSCA and through the DDL. 


\section{4. $\delta$ Orionis Aa-Ab}

MUSCA achieved "first light" on an evening of January 2011 but its full suite of hardware and data reduction pipeline for performing dual-star phasereferencing and binary astrometry was not complete until May/June 2013. The usage of and the results from the pipeline are discussed in this section using observation data of $\delta$ Orionis Aa-Ab.

$\delta$ Orionis Aa-Ab (HR1852; Del Ori) is a close binary star system which has an expected onsky separation of $0.3^{\prime \prime}$, a position angle of $131^{\circ}$ and an orbital period of 201 years (Mason et al., 2009). The primary component is itself a known eclipsing binary ( $\delta$ Orionis Aa1-Aa2) which has an orbital period of 5.7 days (Harvey et al., 1987). The primary component is a spectral type $\mathrm{O}$ star but it is suspected to have yet another tertiary component of the same spectral type (Zasche et al., 2009; Mayer et al., 2010).

$\delta$ Orionis Aa-Ab was observed with MUSCA in 2013 as a target for testing the dual-star phase-referencing mode of the instrument as well as the data reduction pipeline. Table 3 lists the total number of observations attempted on this ternary system. An observation session is considered successful when there are at least 3 sets of bracketed observations of the secondary fringe packet and a bracketed observation consists of a well phase-referenced primary fringe packet and then a well phase-referenced secondary packet and finally another well phase-referenced primary fringe packet. Since the time span for phase-referencing one fringe packet typically last about $10 \mathrm{~min}$, the success rate of an astrometric observation of a binary system with MUSCA is not high because not only is it highly dependent on good atmospheric seeing, but also the duration of the good seeing. Out of 7 consecutive attempts, 2 successful observations were made.

Figures 22(a) and 23(a) show the separations between the primary and the secondary fringe packets of $\delta$ Orionis Aa-Ab plotted over different observation times. For each measurement of the fringe packet separation, 3 possible values are plotted. The first is the fringe separation computed from the phase measurement and the remaining two are the computed fringe separation plus and minus one MUSCA wavelength. The reduced $\chi^{2}$ (shown in Figs. 22(b) and 23(b)) computed during the least square fitting of the fringe separation to the measured differential phases of the fringe packets is used to choose one of the 3 possible values that produces the smallest residual when the fringe separation data are fitted with a binary star model. This is done because the fringe separation obtained from the best least square fit may not be the correct value as it is ambiguous by one MUSCA wavelength due to an astrophysical effect which is discussed in Sec. 7. The selected data points are indicated in the plots with square symbols. In addition to correcting the one MUSCA wavelength ambiguity, the fringe separation measurements are also empirically compensated for errors arising from the phase delay drift effect and the accuracy of the DL metrology using the phase misalignment measured with the primary fringe packets. Table 4 shows

Table 3. Observations of $\delta$ Orionis Aa-Ab.

\begin{tabular}{|c|c|c|c|c|c|c|}
\hline Date $^{\ddagger}$ & Baseline $^{\dagger}$ & Outcome & $\begin{array}{l}\text { Range of } \\
\text { HAs (hr) }\end{array}$ & Calibrators & $\begin{array}{c}\# \text { of MUSCA } \\
\text { scans }^{c}\end{array}$ & $\begin{array}{l}\text { Integration } \\
\text { time }^{\mathrm{c}}(\mathrm{min})\end{array}$ \\
\hline 130105 & N4-S2 & Fail & - & - & - & - \\
\hline 130106 & N1-S2 & Fail & - & - & - & - \\
\hline 130107 & N4-S2 & Success & $0.5-2.0$ & Bellatrix & $\begin{array}{l}\text { Aa: } 500-2000 \\
\text { Ab: } 1000-2000\end{array}$ & $\begin{array}{l}\text { Aa: } 1-4 \\
\text { Aa: } 2-4\end{array}$ \\
\hline 130108 & N4-S2 & Fail & - & - & - & - \\
\hline 130110 & N4-S2 & Fail & - & - & - & - \\
\hline 130111 & N4-S2 & Success & $0.0-2.5$ & Bellatrix & $\begin{array}{l}\text { Aa: } 500-1500 \\
\text { Ab: } 1000-4000\end{array}$ & $\begin{array}{l}\text { Aa: } 1-3 \\
\text { Ab: } 2-9\end{array}$ \\
\hline 130115 & N4-S2 & Fail & - & - & - & - \\
\hline
\end{tabular}



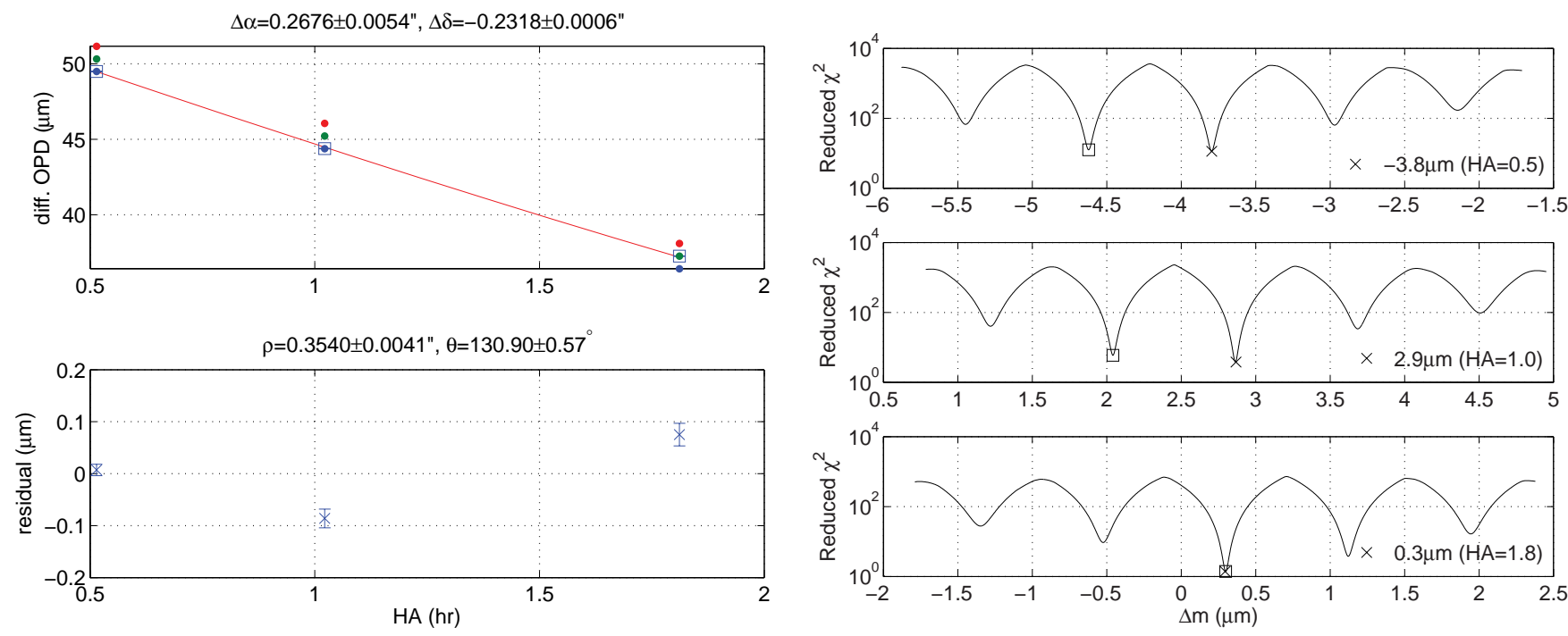

(a)

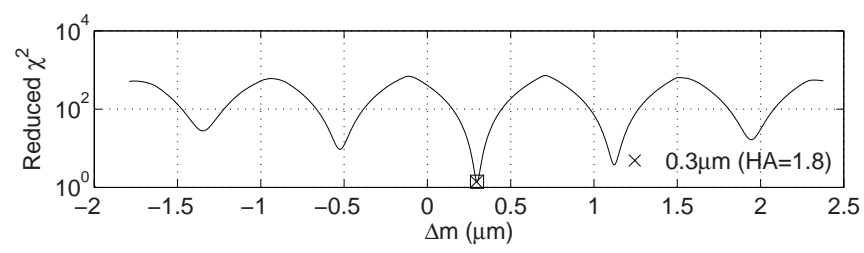

(b)

Fig. 22. Fringe packet separation of $\delta$ Orionis Aa-Ab at different hour angles measured by MUSCA with a 60-m baseline (N4-S2) on 7 Jan 2013. Plots in (b) show the reduced $\chi^{2}$ values of probable apparent separation of the fringe packets. The symbol $\times$ represents the global minimum and the symbol $\square$ represents the most probable value based on the model-fitting in (a).

the phase misalignment between the first and the second primary fringe packet of several bracketed observations. The magnitude of the phase misalignment is within expectation because the DDL is displaced between each measurements. If the fringe packets in the figure are recorded without perturbing the position of the DDL, the phase alignment (not shown in the table) between the fringe packets is measured to be better than $1 / 12$ th of a MUSCA wavelength.

The corrected measurements of the projected separation of the primary and secondary fringe packets, $\tilde{S}+\Delta \tilde{S}$, are used to estimate the position angle, $\theta$, and on-sky separation, $\rho$, of the binary through model-fitting. The model used for parameter extraction is given as,

$$
\begin{aligned}
S & =\Delta \vec{s} \cdot \vec{B} \\
& =\left[\begin{array}{c}
-\cos \delta_{2} \sin \mathrm{HA}_{2}+\cos \delta_{1} \sin \mathrm{HA}_{1} \\
\cos \phi_{\mathrm{LAT}}\left(\sin \delta_{2}-\sin \delta_{1}\right)-\sin \phi_{\mathrm{LAT}}\left(\cos \delta_{2} \cos \mathrm{HA}_{2}-\cos \delta_{1} \cos \mathrm{HA}_{1}\right) \\
\sin \phi_{\mathrm{LAT}}\left(\sin \delta_{2}-\sin \delta_{1}\right)+\cos \phi_{\mathrm{LAT}}\left(\cos \delta_{2} \cos \mathrm{HA}_{2}-\cos \delta_{1} \cos \mathrm{HA}_{1}\right)
\end{array}\right]_{x y z} \cdot\left[\begin{array}{l}
B_{x} \\
B_{y} \\
B_{z}
\end{array}\right]_{x y z},
\end{aligned}
$$

Table 4. Phase stability of the fringes of $\delta$ Orionis Aa in MUSCA.

\begin{tabular}{lcr}
\hline Date & $\begin{array}{c}\text { Hour angle } \\
(\mathrm{Hr})\end{array}$ & $\begin{array}{r}\text { Differential } \\
\text { phase (rad) }\end{array}$ \\
\hline 130107 & 0.5 & $-0.21 \pm 0.06$ \\
& 1.0 & $-0.33 \pm 0.11$ \\
130111 & 1.8 & $0.10 \pm 0.11$ \\
& 0.1 & $-0.51 \pm 0.07$ \\
& 1.0 & $-1.48 \pm 0.07$ \\
& 2.2 & $-1.28 \pm 0.08$ \\
\hline
\end{tabular}

where $S$ is the projected fringe packet separation of two stars, $\vec{B}$ is the vector of the chosen baseline and $\Delta \vec{s}=\hat{s}_{2}-\hat{s}_{1}$ is the difference between pointing vectors of the target and the reference stars. Both vectors are resolved into their components in a rectangular coordinate system, taking the ground as the frame of reference. The $x$-axis points to the East, $y$ axis points to the North and $z$-axis points to zenith. The celestial coordinates, $\left(\mathrm{HA}_{1}, \delta_{1}\right)$ and $\left(\mathrm{HA}_{2}, \delta_{2}\right)$, define the location of the primary and secondary 
stars in the sky where $\delta_{2}=\delta_{1}+\rho \cos \theta$ and $\mathrm{HA}_{2}=$ $\mathrm{HA}_{1}-\rho \sin \theta$. The symbols $\rho$ and $\theta$ represent the onsky separation and position angle of the two stars in a polar coordinate system. Although the uncertainty in the position of the primary star translates to an uncertainty in projected fringe packet separation, the value is extremely small and negligible. For example, through computer simulation, it was found that a standard deviation of $0.5^{\prime \prime}$ in both axes of the coordinate system produces a standard deviation of less than $1 \mathrm{~nm}$ in projected fringe packet separation measurements, even at an unfavorable pointing angle and with a long $(160 \mathrm{~m})$ baseline. By unfavorable pointing angle, we mean that the angle subtended by the pointing vector, $\hat{s}$, and the baseline vector, $\vec{B}$, is very small. This means the parameters extracted from fitting the model to the measured projected separation are insensitive to the absolute position of the primary star if the accuracy is better than $0.5^{\prime \prime}$. This value is large compared to the proper motion of stars suitable for MUSCA observation which are mainly less than $0.25^{\prime \prime}$. Finally the symbol $\phi_{\mathrm{LAT}}$ in the
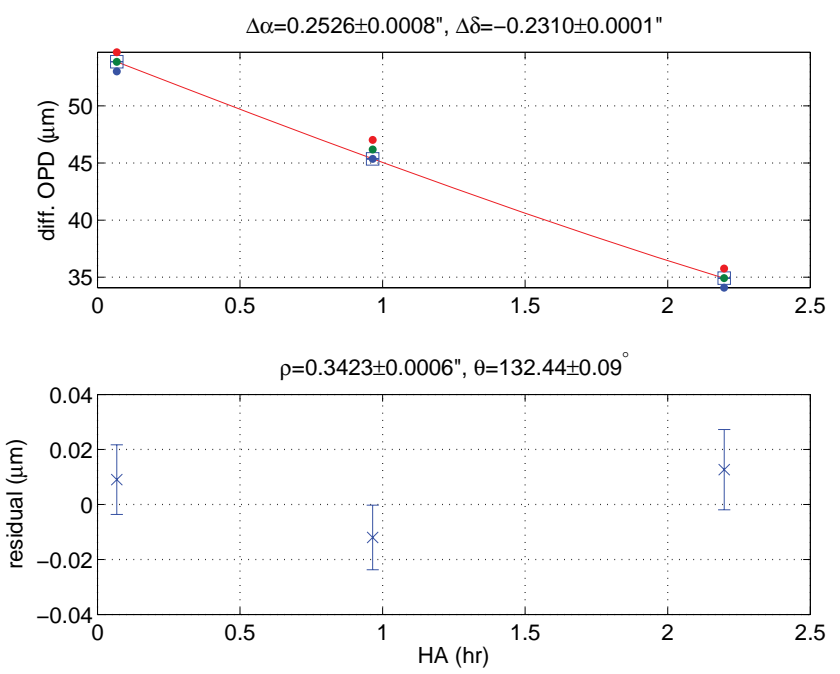

(a) above equation represents the geographical latitude of SUSI.

An alternative model described by Rizzuto et al. (2013) can also be used. However it may not be suitable for high-precision narrow-angle astrometry of stars separated by more than $3^{\prime \prime}$ and/or using baselines longer than $160 \mathrm{~m}$ because the approximation in $\Delta \vec{s} \cdot \vec{B}$ may exceed $10 \mathrm{~nm}$. A discussion of this approximation error is included in App. A.

The fitting of the binary star model to the fringe packet separation measurements from the two observations have residuals of less than $\sim 100 \mathrm{~nm}$, a magnitude which is consistent with the intrinsic misalignment observed in the internal phase stability test discussed earlier. The residuals of the fits are plotted in the lower panels of Figs. 22(a) and 23(a).

The relative astrometry of $\delta$ Orionis $\mathrm{Aa}-\mathrm{Ab}$ obtained from the two observations is summarized in Table 5. Since the position of the vernal equinox, which is the origin of the equatorial coordinate system, changes over time due to perturbation in the Earth's rotation axis, the astrometric
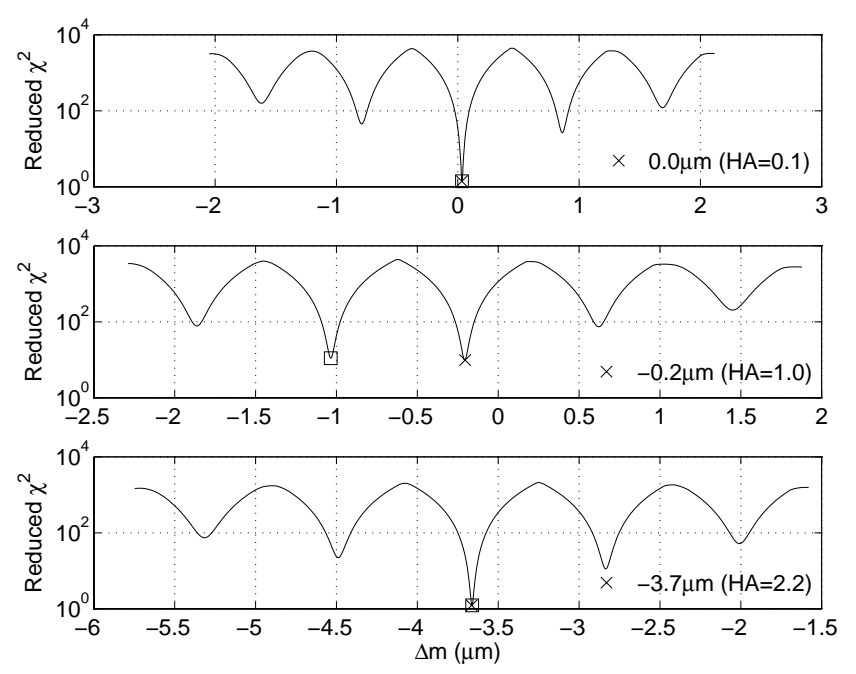

(b)

Fig. 23. Similar to Fig. 22 but with data taken on 11 Jan 2013.

Table 5. Relative astrometry of $\delta$ Orionis Aa-Ab.

\begin{tabular}{lcccc}
\hline Parameter & \multicolumn{2}{c}{ As fitted } & \multicolumn{2}{c}{$\mathrm{Eq}=\mathrm{J} 2000$} \\
\hline Epoch & 2013.0178 & 2013.0287 & 2013.0178 & 2013.0287 \\
$\Delta \alpha_{\mathrm{Aa}, \mathrm{Ab}}(\mathrm{mas})$ & $267.6 \pm 5.4$ & $252.6 \pm 0.8$ & $267.3 \pm 5.4$ & $252.3 \pm 0.8$ \\
$\Delta \delta_{\mathrm{Aa}, \mathrm{Ab} \text { (mas) }}$ & $-231.8 \pm 0.6$ & $-231.0 \pm 0.1$ & $-232.1 \pm 0.6$ & $-231.3 \pm 0.1$ \\
$\rho_{\mathrm{Aa}, \mathrm{Ab}}(\mathrm{mas})$ & $354.0 \pm 4.1$ & $342.3 \pm 0.6$ & $354.0 \pm 4.1$ & $342.3 \pm 0.6$ \\
$\theta_{\mathrm{Aa}, \mathrm{Ab}}\left(^{\circ}\right)$ & $130.90 \pm 0.57$ & $132.44 \pm 0.09$ & $130.97 \pm 0.88$ & $132.51 \pm 0.14$ \\
$\chi_{\mathrm{R}}^{2}$ & 35.2 & 2.3 & - & - \\
\hline
\end{tabular}


Table 6. Comparison of $\delta$ Orionis Aa-Ab astrometry.

\begin{tabular}{lccccc}
\hline Source & $\begin{array}{c}\text { Epoch } \\
(+2000)\end{array}$ & $\begin{array}{c}\theta \\
\left(^{\circ}\right)\end{array}$ & $\begin{array}{c}\delta \theta \\
\left(^{\circ}\right)\end{array}$ & $\begin{array}{c}\rho \\
\left(^{\prime \prime}\right)\end{array}$ & $\begin{array}{c}\delta \rho \\
(\mathrm{mas})\end{array}$ \\
\hline WDS & 13.1311 & 131.0 & - & 0.3273 & 0.2 \\
Ephemeris $^{*}$ & 13.1000 & 130 & - & 0.31 & - \\
This work & 13.0287 & 132.51 & 0.14 & 0.3423 & 0.6 \\
& 13.0178 & 130.97 & 0.88 & 0.3540 & 4.1 \\
MA2010 $^{\dagger}$ & 08.0449 & 132.66 & 0.47 & 0.325 & 1.6 \\
\hline
\end{tabular}

* Based on a grade 4 (preliminary) orbit from ORB6.

†Maíz Apellániz (2010).

parameters in the table are also expressed in an equatorial coordinate system that is based on equinox $2000^{(\mathrm{b})}$ for comparison with astrometry performed with other techniques and published values in the literature. The coordinate transformation involved took the effect of precession and nutation of the Earth's rotation axis into account.

For comparison, measurements of the separation and position angle of $\delta$ Orionis $\mathrm{Aa}-\mathrm{Ab}$ obtained from the Fourth Catalog of Interferometric Measurements of Binary Stars ${ }^{(c)}$ (INT4) and the Washington Visual Double Star ${ }^{(d)}$ (WDS) catalog are listed in Table 6 . The measurements obtained in this paper agree only moderately well (to within $\sim 1^{\circ}$ in position angle and $\sim 0.02^{\prime \prime}$ in binary separation) with measurements from the WDS catalog. An ephemeris of the secondary component computed based on parameters from the Sixth Catalog of Orbits of Visual Binary Stars ${ }^{(e)}$ (ORB6) is also included for comparison.

\section{Discussion}

Despite the expected alignment of the fringe phases of better than $1 / 4$ th of a wavelength $(<1.5 \mathrm{rad})$, the primary fringe packet registers discrete jumps of about one MUSCA wavelength in its relative position. Plots in Fig. 24 show the jumps at hour angles (HA) of $\sim 0.275$ and $\sim 1.35$ respectively. The jumps are not related to the accuracy of the dual laser metrology because fringe packets in the figure are recorded without any displacement of DDL in between. Furthermore, these jumps occur within

\footnotetext{
${ }^{\mathrm{b}}$ The origin of the coordinate system is centered on the position of the vernal equinox in the year 2000 .

${ }^{c}$ http://ad.usno.navy.mil/wds/int4.html.

${ }^{\mathrm{d}}$ http://ad.usno.navy.mil/wds.

${ }^{\mathrm{e}}$ http://ad.usno.navy.mil/wds/orb6.html.
}

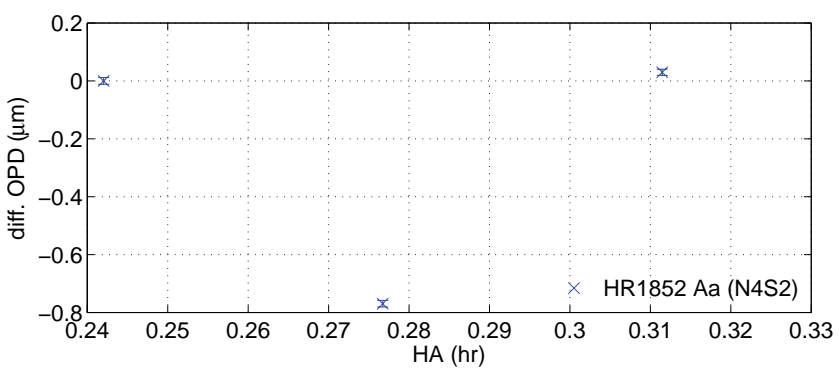

(a)

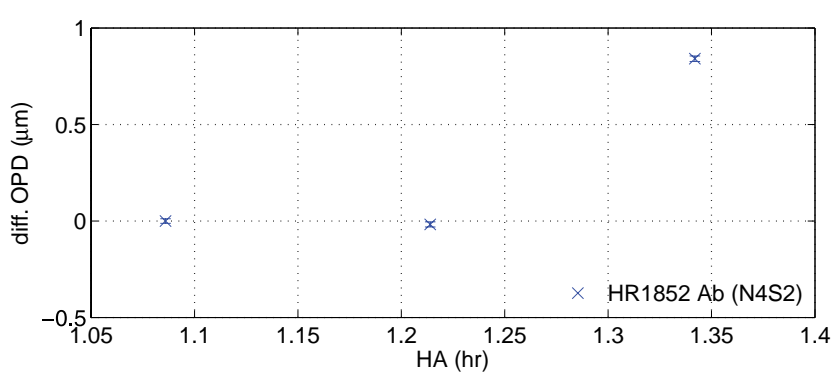

(b)

Fig. 24. Relative fringe packet position of $\delta$ Orionis Aa1 measured at different times showing jumps of one MUSCA wavelength occasionally due to the presence of an unresolved fringe packet of $\delta$ Orionis Aa2.

time intervals that are too short for an unresolved secondary fringe packet to drift by the same amount.

The origin of the fringe packet position jump observed can be traced back to the method the phase delay was computed from the PAVO interferograms. Phase delays are computed from group delays which in turn are estimated from the position of the peak of the Fourier transform of the complex visibility of the fringes across the PAVO bandpass. The detailed description of this algorithm is given in Sec. 4.1.1. A computer simulated example plot of the Fourier transform, $\hat{\mathbf{q}}$ in Eq. (13), is shown in the top panel of Fig. 25(a). In the example, the fringes belong to a single star and the phase delay (indicated by the symbol $z$ in the figure) is zero (within the uncertainty of the algorithm). When fringes from a secondary star are introduced into the simulation, the phase delay of the fringes from the primary star is altered depending on the fringe separation and the visibility of the secondary star fringes. Similar example plots of $\hat{\mathbf{q}}$ when PAVO interferograms consist of fringes from two stars are shown in the middle and bottom panels of Fig. 25(a). Different values of fringe separations, indicated by the symbol $S$ in the legend, are chosen in order to illustrate the effect of additional fringes 

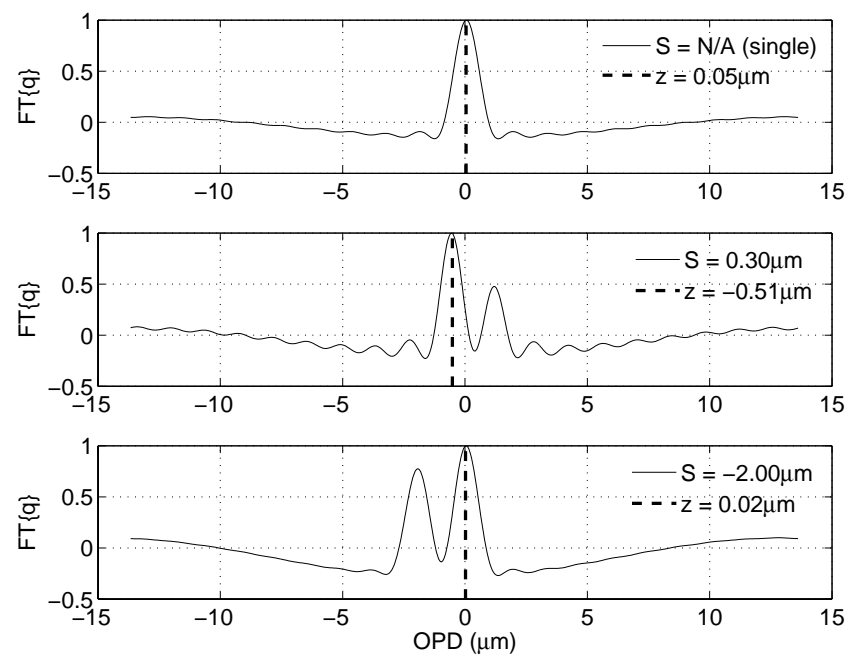

(a)

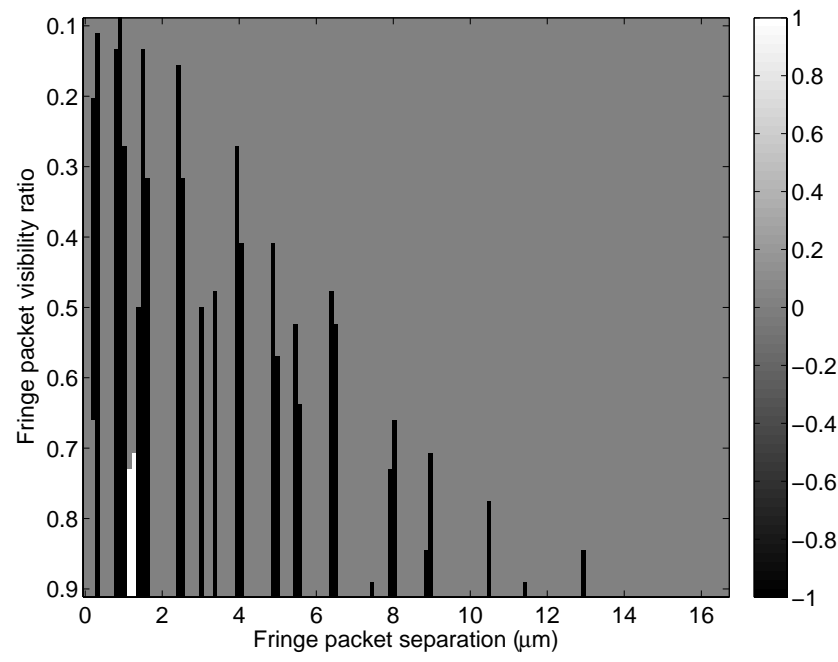

(b)

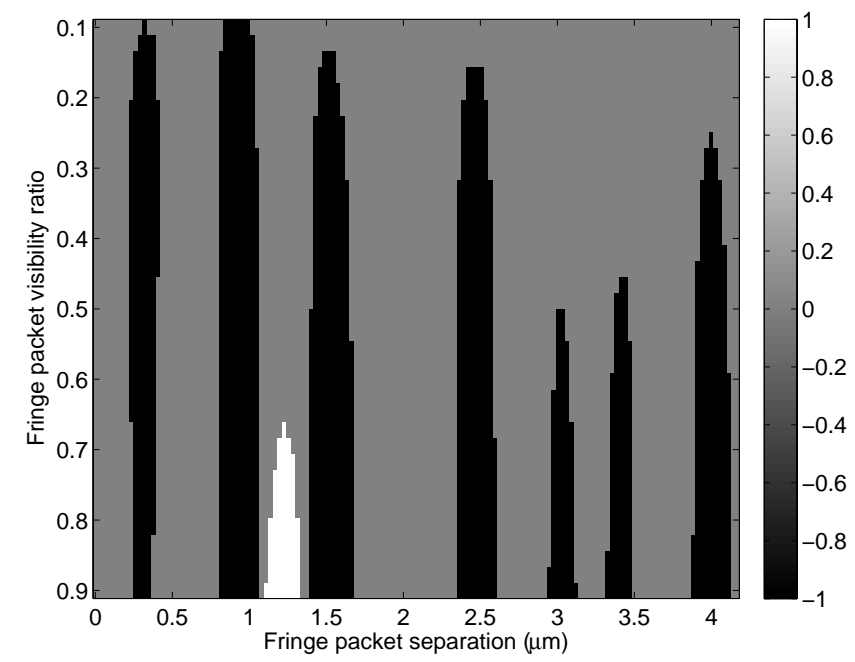

(c)

Fig. 25. The plots in (a) show the Fourier transform of the complex visibility, a variable computed in the data reduction pipeline to obtain the phase delay of a set of fringes from the location of the peak in the plots (see Eq. (13)). The addition of a second set of fringes at the right fringe separation can artificially shift the peak, thereby resulting in an incorrect phase delay computed for the first. Due to this error, the relative phase delay of the primary fringes between the middle and bottom panels of (a) will wrongly be computed as one MUSCA wavelength despite them being kept unchanged. Plots in (b) and (c) show the relative phase delay error in MUSCA wavelengths for various fringe separations and visibility ratios (primary vs. secondary set of fringes).

on the phase delay of the primary. The ratio of the visibility of the two sets of fringes in the plots is chosen to be 0.9. The position of the peak of $\hat{\mathbf{q}}$ in the middle panel clearly shows the phase delay deviates from zero. If the phase delay deviates by more than half of a MUSCA wavelength, then the fringe separation computed between the current and a reference MUSCA fringe packet will register a jump of one MUSCA wavelength, a phenomenon observed in Fig. 24. The magnitude of the jump for a range of primary-secondary fringe packet separations and visibility ratios is shown in Figs. 25(b) and $25(\mathrm{c})$. The images in the figure show various locations where phase delay jumps can occur and the grayscale of the image shows the magnitude of the jump in MUSCA wavelengths. Figure 25(c) is a zoomed in version of (b). The latter also shows that the probability of a phase delay jump occurring is zero when the fringe packet separation is larger than $\sim 15 \mu \mathrm{m}$ which is half of the coherence length of one 
PAVO spectral channel. This is expected because the fringe packets will no longer overlap each other at that separation.

The PAVO $V^{2}$ data from the successful observations were analyzed to evaluate the structure of the primary component. The $V^{2}$ data showed no amplitude modulation from the secondary $(\delta$ Orionis $\mathrm{Ab})$ fringe packet because the separation of the primary-secondary fringe packet pair $(\sim 35-55 \mu \mathrm{m})$ is larger than the width of a single fringe packet and hence there is little overlap. However, the presence of incoherent flux from the secondary component limits the maximum $V^{2}$ of the primary to $\sim 0.66$. Taking this into account, the data when fitted with a simple uniform disk (UD) model suggests that the primary star has an average angular diameter (over two observations) of $1.05 \pm 0.02$ mas. The data and the fitted models are shown in Fig. 26. The estimated UD diameter corresponds to $\sim 48$ solar radii $\left(\mathrm{R}_{\odot}\right)$ at $\sim 212 \mathrm{pc}(\mathrm{com}-$ puted from parallax measurement (van Leeuwen, 2007)). This is consistent with the estimated diameters of the eclipsing components ( $\delta$ Orionis Aa1 and $\delta$ Orionis Aa2), which consist of an O-type and a B-type star and have estimated angular diameters of $\sim 30 \mathrm{R}_{\odot}$ and $\sim 10 \mathrm{R}_{\odot}$ respectively (Mayer et al., 2010). Due to such astrophysical structures, the phase of the primary ( $\delta$ Orionis Aa) fringe packet changes over time as the asymmetry of the structure rotates with respect to the baseline of the interferometer. For the sake of estimating the magnitude of the fringe packet separation of the eclipsing components, the binary separation is assumed to be of the order of the estimated UD diameter, i.e. $\approx 1$ mas, and the position angle is assumed to be parallel

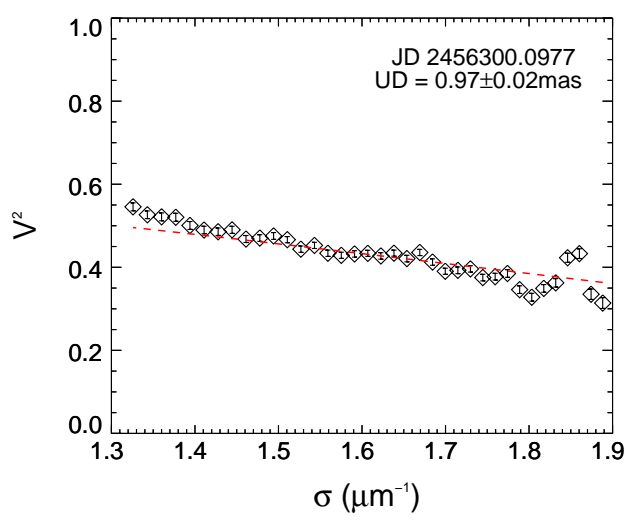

(a) to the baseline. In this case, the fringe packet separation observed with a $60 \mathrm{~m}$ baseline (N4-S2) is $\lesssim 0.3 \mu \mathrm{m}$. Given the sensitivity of the phase delay computation algorithm to a secondary fringe packet at this separation, it is likely that the jumps in the relative position of the primary fringe packets are caused by the presence of an unseen fringe packet of the tertiary component ( $\delta$ Orionis Aa2). In general, the double fringe packet may not be easily distinguished from the data if the tertiary fringe packet is partially resolved or buried within the primary fringe packet of a binary star and especially when the integration time is long enough for the tertiary to move by more than one fringe. The systematic error in the relative positions of the primary fringe packet also affects the estimated fringe packet separation because the fringes of the secondary ( $\delta$ Orionis $\mathrm{Ab}$ ) component were phase-referenced to the fringes of the primary. The reduced $\chi^{2}$ values from a least square fit analysis carried out to determine the relative position of fringe packets can be used to estimate the correct position in such circumstances.

The binary separations in the declination axis $\left(\Delta \delta_{\mathrm{Aa}, \mathrm{Ab}}\right)$ measured on the two different nights of observation differ by less than 1 mas, which is consistent with the dimension of the angular diameter of the primary component or the binary separation of $\delta$ Orionis Aa1-Aa2. Since the two observations were 3.9 days apart, this consistency is also supported by the orbital period of the eclipsing binary (5.7 days) which suggests the photo center of the primary component could be on opposite sides of the uniform disk on the two nights of observation. On the other hand, the binary separations in the

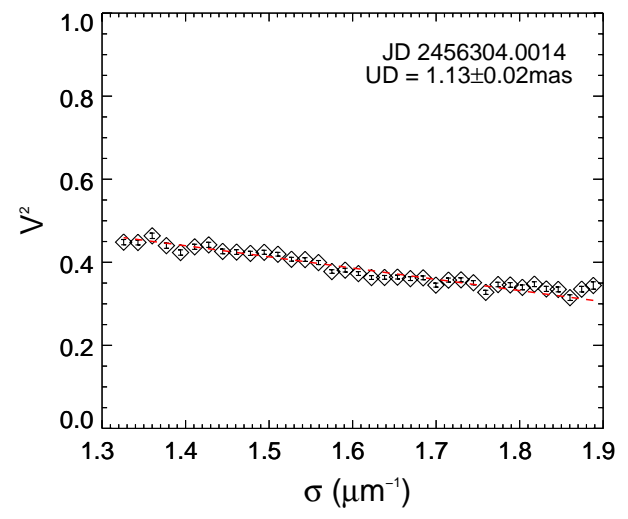

(b)

Fig. 26. Calibrated $V^{2}$ data of $\delta$ Orionis Aa obtained with PAVO and a 60-m baseline (N4-S2). Uniform disk (UD) models that best fit the data are plotted as dashed lines and the UD diameters from the fits are indicated in the plots. 
right ascension axis $\left(\Delta \alpha_{\mathrm{Aa}, \mathrm{Ab}}\right)$ measured on the two different nights differ by more than 10 mas. This discrepancy cannot be an effect of the orbital motion of the secondary component $(\mathrm{Ab})$ around the primary (Aa) because the estimated orbital period for the pair is 201 years (Mason et al., 2009) and the estimated motion within $\sim 4$ days is $\ll 0.1$ mas. It is also unlikely to be an effect of narrowangle baseline misalignment because in order to produce the astrometric uncertainty $(\sim 10$ mas over $\sim 260$ mas of projected separation) obtained from the data analysis the East-West component of the narrow-angle baseline on the two nights must differ by $\sim 30 \mathrm{~cm}$, a dimension which is larger than the diameter of the main siderostat mirror. Therefore, the discrepancy in the right ascension axis suggests that there are systematic errors on top of the known phase misalignment which are not accounted for in the data analysis.

At the time of writing, none of the close binary systems observed for performance testing, either with MUSCA or PAVO, consists of just 2 component stars. More MUSCA observations, especially with targets that do not have spectroscopic companions, and data analyses are needed to understand the source of the systematic errors in MUSCA which may or may not be related to the differential delay line and the dual-laser metrology. In that aspect, the low success rate of a MUSCA observation is a challenge that must be overcome especially for future observation campaigns.

\section{Conclusion}

This paper has documented a successful attempt at high-precision narrow-angle astrometry by means of optical long baseline interferometry. The astrometric facility setup at SUSI is now operational and its first generation results have been demonstrated. The PAVO+MUSCA setup in SUSI is the first dual beam combiner that is capable of performing dual-star phase-referencing interferometry at visible wavelengths and it achieves that through a novel post-processing approach. However, the current best astrometric precision of MUSCA is in the order of $100 \mu$ as in the declination axis while the precision in the right ascension axis is uncertain as the source of the large discrepancy between two measurements is currently unknown. Further observations and improvements are needed to ascertain and optimize the performance of the facility and the precision of its astrometry. The detection principles outlined in this paper could also be applied to interferometers at other sites, especially a potential astrometric interferometer located on the high Antarctic plateau.

\section{Acknowledgments}

This research was supported by the Australian Research Council's Discovery Project funding scheme. Y. K. was supported by the University of Sydney International Scholarship (USydIS) and would like to acknowledge the helpful discussions with Vicente Maestro. The authors would also like to acknowledge the use of the electronic bibliography maintained by NASA/ADS system, the Washington Double Star Catalog maintained by the U.S. Naval Observatory and the SIMBAD/VizieR database maintained by CDS, Strasbourg, France.

\section{Appendix A}

The model in Sec. 5 was chosen over an alternative model described by (Rizzuto et al., 2013). The latter is worth a brief discussion even though it is not used in this work as it is suitable for less precise narrow-angle astrometry. The model uses a coordinate system that takes the sky as the frame of reference. In order to differentiate the frame of reference from that used for this work, the axes of the coordinate system are labeled $u, v$ and $w$. The $u$ axis points to the celestial East, the $v$-axis points to the celestial North and the $w$-axis points to towards the sky. The model is given as,

$$
\begin{aligned}
S & =\vec{\rho} \cdot \vec{B}_{p} \\
& =\left[\begin{array}{c}
\rho \sin \theta \\
\rho \cos \theta \\
0
\end{array}\right]_{u v w} \cdot\left[\begin{array}{c}
B_{u} \\
B_{v} \\
0
\end{array}\right]_{u v w},
\end{aligned}
$$

where $\vec{\rho}$ is a vector defining the separation of two stars in the two-dimensional $u v$-plane of the sky and $\vec{B}_{p}$ is the component of $\vec{B}$ projected onto the same plane. Since $\vec{B}$ is commonly quoted in the ground ( $x y z)$ frame because it is more natural to an observer on the ground, it can be converted into the sky (uvw) frame as shown,

$$
\left[\begin{array}{l}
B_{u} \\
B_{v} \\
B_{w}
\end{array}\right]_{u v w}=\mathbf{T}\left[\begin{array}{l}
B_{x} \\
B_{y} \\
B_{z}
\end{array}\right]_{x y z},
$$


where $\mathbf{T}$ is a matrix for coordinate transformation (Fomalont \& Wright, 1974), which is given as,

$$
\mathbf{T}=\left[\begin{array}{ccc}
\cos \mathrm{HA} & \sin \phi_{\mathrm{LAT}} \sin \mathrm{HA} & \cos \phi_{\mathrm{LAT}} \sin \mathrm{HA} \\
\sin \delta \sin \mathrm{HA} & \cos \phi_{\mathrm{LAT}} \cos \delta+\sin \phi_{\mathrm{LAT}} \sin \delta \cos \mathrm{HA} & \sin \phi_{\mathrm{LAT}} \sin \delta-\cos \phi_{\mathrm{LAT}} \sin \delta \cos \mathrm{HA} \\
-\cos \delta \sin \mathrm{HA} & \cos \phi_{\mathrm{LAT}} \sin \delta-\sin \phi_{\mathrm{LAT}} \cos \delta \cos \mathrm{HA} & \sin \phi_{\mathrm{LAT}} \sin \delta+\cos \phi_{\mathrm{LAT}} \cos \delta \cos \mathrm{HA}
\end{array}\right] .
$$

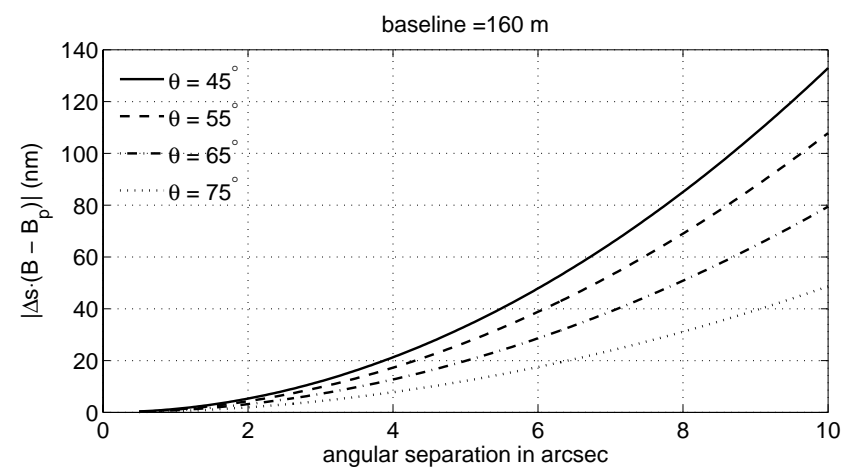

Fig. A.1. The plot shows the optical path length contributed by $\Delta \vec{s} \cdot \vec{B}_{p}-\Delta \vec{s} \cdot \vec{B}$. The angle $\theta$ in the legend represents the angle between $\hat{s}_{1}$ and $\vec{B}$.

The RHS of Eq. (A.1) is an approximation of $\Delta \vec{s} \cdot \vec{B}$ and therefore the accuracy of the parameters extracted from the model could be systematically less than parameters extracted from our model (Eq. 28). The calculated projected separation using Eq. (A.1) could be off by as much as $6 \mathrm{~nm}$ under certain conditions. This undesirable value translates to more than $10 \mu$ as of astrometric error if the baseline of the interferometer is $100 \mathrm{~m}$ or less. The factors affecting the approximation error are given as,

$$
\begin{aligned}
\Delta \vec{s} \cdot \vec{B} & =\Delta \vec{s} \cdot \vec{B}_{p}-2\left|\hat{s}_{1} \cdot \vec{B}\right| \sin ^{2} \frac{\Delta \theta}{2} \\
& \approx \vec{\rho} \cdot \vec{B}_{p}-2\left|\hat{s}_{1} \cdot \vec{B}\right| \sin ^{2} \frac{\Delta \theta}{2}
\end{aligned}
$$

where $\Delta \theta$ is the angle subtended by the pointing vectors of the primary and secondary stars. In the narrow-angle regime, $\Delta \theta \approx|\Delta \vec{s}|$ and $\Delta \vec{s} \approx \vec{\rho}$. The approximation error of the latter is negligible even at long baseline $(>100 \mathrm{~m})$. On the contrary, the plot in Fig. A.1 shows that the approximation error of $\Delta \vec{s} \cdot \vec{B}_{p} \approx \Delta \vec{s} \cdot \vec{B}$ is negligible only for very closely separated stars. In summary, this alternative model is still (just) suitable for MUSCA, which has a FOV of $<2^{\prime \prime}$, but may not be for other instruments with a wider FOV.

\section{References}

Antoci, V., Handler, G., Grundahl, F. et al., 2013, MNRAS, 435, 1563-1575, doi:10.1093/mnras/stt1397.

Colavita, M., Wallace, J., Hines, B. et al., 1999, ApJ, 510, 505, doi:10.1086/306579.

Colavita, M. M. 1992, Phase referencing for stellar interferometry, In European Southern Observatory Conference and Workshop Proceedings, eds. Beckers, J. M. \& Merkle, F., p. 845.

Daendliker, R., Thalmann, R. \& Prongue, D. 1988, Optics Letters, 13, 339, doi:10.1364/OL.13.000339.

Davis, J., Ireland, M. J., Chow, J. et al., 2007, Publications of the Astronomical Society of Australia, 24, 138, doi:10.1071/AS07016.

Davis, J., Tango, W. J., Booth, A. J. et al., 1999a, MNRAS, 303, 773, doi:10.1046/j.1365-8711.1999.02269.x.

Davis, J., Tango, W. J., Booth, A. J., Thorvaldson, E. D. \& Giovannis, J. 1999b, MNRAS, 303, 783, doi:10.1046/ j.1365-8711.1999.02270.x.

Delplancke, F. 2008, New Astronomy Review, 52, 199, doi:10.1016/j.newar.2008.04.016.

Fomalont, E. B. \& Wright, M. C. H. 1974, Interferometry and aperture synthesis, In Verschuur, G. L., Kellermann, K. I., eds., Galactic and Extra-Galactic Radio Astronomy (Springer), p. 256.

Harvey, A. S., Stickland, D. J., Howarth, I. D. \& Zuiderwijk, E. J. 1987, The Observatory, 107, 205.

Hummel, C. A., Mozurkewich, D., Benson, J. A. \& Wittkowski, M. 2003, Coherent integration using phase bootstrapping. In Proc. SPIE, 4838 ed. W. A. Traub, p. 1107.

Ireland, M. J., Mérand, A., ten Brummelaar, T. A. et al., 2008, Sensitive visible interferometry with PAVO, Proc. SPIE, 7013, doi:10.1117/12.788386.

Kok, Y., Ireland, M. J., Robertson, J. G. et al., 2013a, Appl. Opt., 52, 2808, doi:10.1364/AO.52.002808.

Kok, Y., Maestro, V., Ireland, M. J., Tuthill, P. G. \& Robertson, J. G. 2013b, Experimental Astronomy, 36, 195, doi:10.1007/s10686-013-9331-8.

Lane, B. F. \& Colavita, M. M. 2003, AJ, 125, 1623, doi:10.1086/367793.

Lane, B. F. \& Muterspaugh, M. W. 2004, ApJ, 601, 1129, doi:10.1086/380760.

Lawson, P. R. 1994, PASP, 106, 917, doi:10.1086/133461.

Maíz Apellániz, J. 2010, AESA, 518, A1, doi:10.1051/0004$6361 / 201014409$.

Mason, B. D., Hartkopf, W. I., Gies, D. R., Henry, T. J. \& Helsel, J. W. 2009, AJ, 137, 3358, doi:10.1088/0004$6256 / 137 / 2 / 3358$.

Mayer, P., Harmanec, P., Wolf, M., Božić, H. \& Šlechta, M. 2010, A $\mathscr{G} A$, 520, A89, doi:10.1051/0004-6361/200913796. 
Muterspaugh, M. W., Lane, B. F., Kulkarni, S. R. et al., 2010, AJ, 140, 1579, doi:10.1088/0004-6256/140/6/1579.

Quirrenbach, A., Mozurkewich, D., Buscher, D. F., Hummel, C. A. \& Armstrong, J. T. 1994, A\&\&A, 286, 1019.

Rizzuto, A. C., Ireland, M. J., Robertson, J. G. et al., 2013, MNRAS, 436, 1694, doi:10.1093/mnras/stt1690.

Robertson, J. G., Ireland, M. J., Tango, W. J. et al., 2010, Instrumental developments for the Sydney University Stellar Interferometer, Proc. SPIE, 7743, doi:10.1117/ 12.856557.

Robertson, J. G., Ireland, M. J., Tango, W. J. et al., 2012, Science and technology progress at the Sydney University Stellar Interferometer, Proc. SPIE, 8445, doi:10.1117/12.924946.

Scargle, J. D. 1989, ApJ, 343, 874.

Schuhler, N., Salvadé, Y., Lévêque, S., Dändliker, R. \& Holzwarth, R. 2006, Optics Letters, 31, 3101, doi:10.1364/OL.31.003101.
Shao, M. \& Colavita, M. 1992, A\& A, 262, 353.

Tango, W. J. 1990, Appl. Opt., 29, 516, doi:10.1364/ AO.29.000516.

ten Brummelaar, T. A. \& Tango, W. J. 1994, Experimental Astronomy 4, 297, doi:10.1007/BF01580788.

Tuthill, P., Davis, J., Ireland, M. et al., 2008, The SUSI instrument: new science and technology, Proc. SPIE, 7013, doi:10.1117/12.787707.

van Leeuwen, F. 2007, A\&SA, 474, 653, doi:10.1051/00046361:20078357.

Woillez, J., Akeson, R., Colavita, M. et al., 2010, ASTRA: astrometry and phase-referencing astronomy on the Keck interferometer, Proc. SPIE, 7734, doi:10.1117/12.857740.

Woillez, J. \& Lacour, S. 2013, ApJ, 764, 109, doi:10.1088/ 0004-637X/764/1/109.

Zasche, P., Wolf, M., Hartkopf, W. I. et al., 2009, AJ, 138, 664, doi:10.1088/0004-6256/138/2/664. 